Supporting Information for:

\title{
Structures of three di-substituted [13]-macrodilactones reveal effects of substitution on macrocycle conformation
}

Kelli M. Rutledgea, Caleb Griesbacha, Brandon Q. Mercadob*, Mark W. Peczuha ${ }^{a^{*}}$

${ }^{a}$ Department of Chemistry, University of Connecticut, 55 N. Eagleville Road, U3060, Storrs, CT 06269, USA

${ }^{\mathrm{b}}$ Department of Chemistry, Yale University, PO Box 208107, New Haven, Connecticut 06520-8107, USA

Total number of pages: 26

Table of Contents

\begin{tabular}{|c|c|}
\hline Item & Page(s) \\
\hline $\begin{array}{l}\text { Figure S1. The fragments used to search the Cambridge Structure Database (CSD } \\
2017+4 \text { updates). The dashed lines highlight the 3D contacts defined within } \\
\text { ConQuest. }\end{array}$ & 2 \\
\hline Figure S2. Close contact in compound I & 2 \\
\hline $\begin{array}{l}\text { Figure S3. The distances (DIST1 in } \AA \text { ) for } 3796 \text { structures with intermolecular phenyl } \\
\text { bromine interactions (CSD } 2017+4 \text { updates). }\end{array}$ & 3 \\
\hline Figure S4. Close contact in compound II & 3 \\
\hline $\begin{array}{l}\text { Figure S5. The distances (DIST1 in } \AA \text { ) for } 2486 \text { structures with intermolecular phenyl } \\
\text { bromine interactions (CSD } 2017+4 \text { updates). }\end{array}$ & 4 \\
\hline Figure S6. Close contact in compound III & 4 \\
\hline $\begin{array}{l}\text { Figure } 57 \text {. The distances (DIST1 in } \AA \text { ) for } 88 \text { structures with intermolecular phenyl } \\
\text { bromine interactions (CSD } 2017+4 \text { updates). }\end{array}$ & 5 \\
\hline Table S1. Substitution patterns and refcodes for [13]-macrodilactones & 6 \\
\hline Table S2. Aspect ratios of compounds in Figure 3 from X-ray crystallographic data & 7 \\
\hline $\begin{array}{l}\text { Table S3. RMSD }(\AA) \text { values comparing X-ray and DFT optimized structures for the } \\
\text { core ring atoms of the [13]-macrodilactones }\end{array}$ & 8 \\
\hline Table S4. Torsion angles, bond angles, and bond lengths of compound "a" ring atoms. & $9-10$ \\
\hline $\begin{array}{l}\text { Table S5. Torsion angles, bond angles, and bond lengths of compound " } g \text { " II ring } \\
\text { atoms. }\end{array}$ & $10-11$ \\
\hline Table S6. Torsion angles, bond angles, and bond lengths of compound "e" ring atoms. & $11-12$ \\
\hline $\begin{array}{l}\text { Table S7. Torsion angles, bond angles, and bond lengths of compound " } h \text { "I ring } \\
\text { atoms. }\end{array}$ & $12-13$ \\
\hline $\begin{array}{l}\text { Table S8. Torsion angles, bond angles, and bond lengths of compound " } k \text { " III ring } \\
\text { atoms. }\end{array}$ & $13-14$ \\
\hline${ }^{1} \mathrm{H}$ and ${ }^{13} \mathrm{C}$ spectra for new compounds & $15-26$ \\
\hline
\end{tabular}



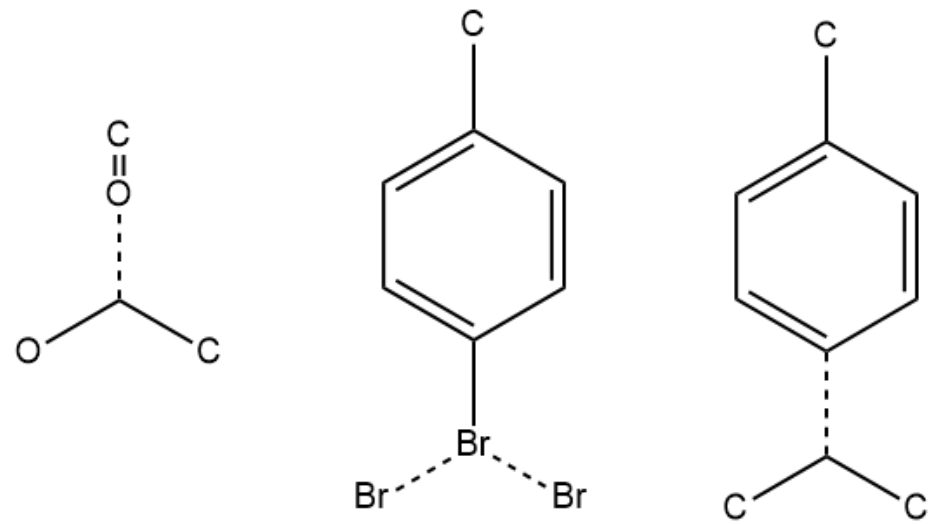

Fragment for 1 Fragment for 2 Fragment for $\mathbf{3}$

Figure S1. The fragments used to search the Cambridge Structure Database (CSD $2017+4$ updates). The dashed lines highlight the 3D contacts defined within ConQuest.

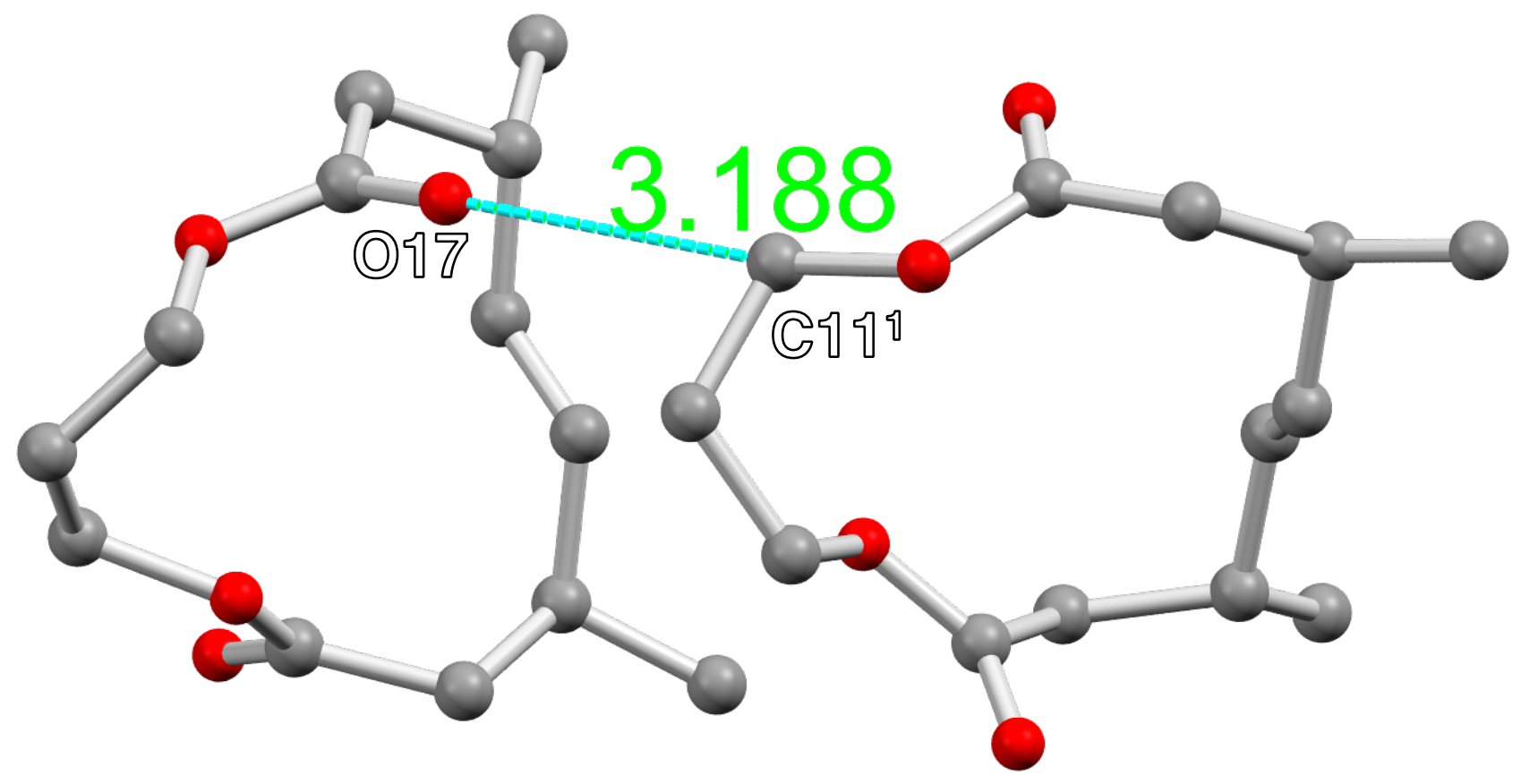

Figure S2. Interaction identified in I. Operator 1 is $x, \frac{3}{2}-y,-\frac{1}{2}+z$. 


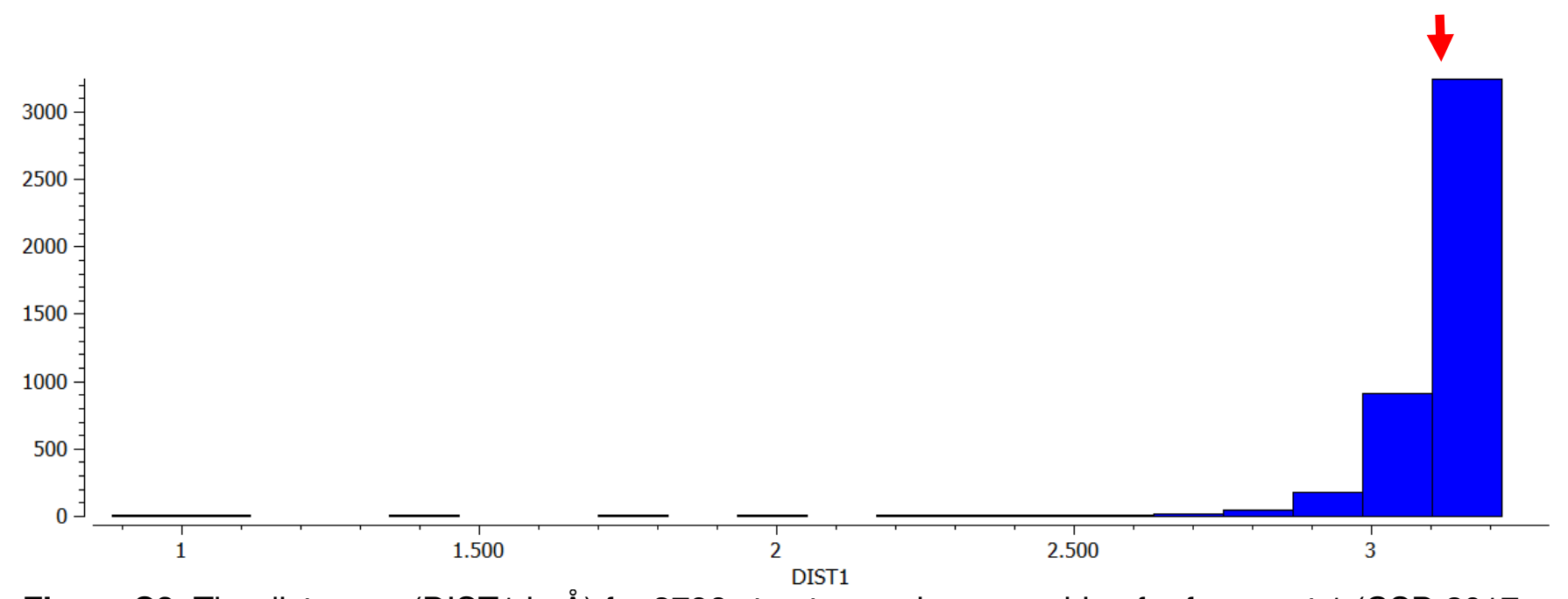

Figure S3. The distances (DIST1 in $\AA$ ) for 3796 structures when searching for fragment 1 (CSD 2017 + 4 updates). The red arrow identifies were the distance identified in $\mathbf{S 2}$ falls in the histogram.

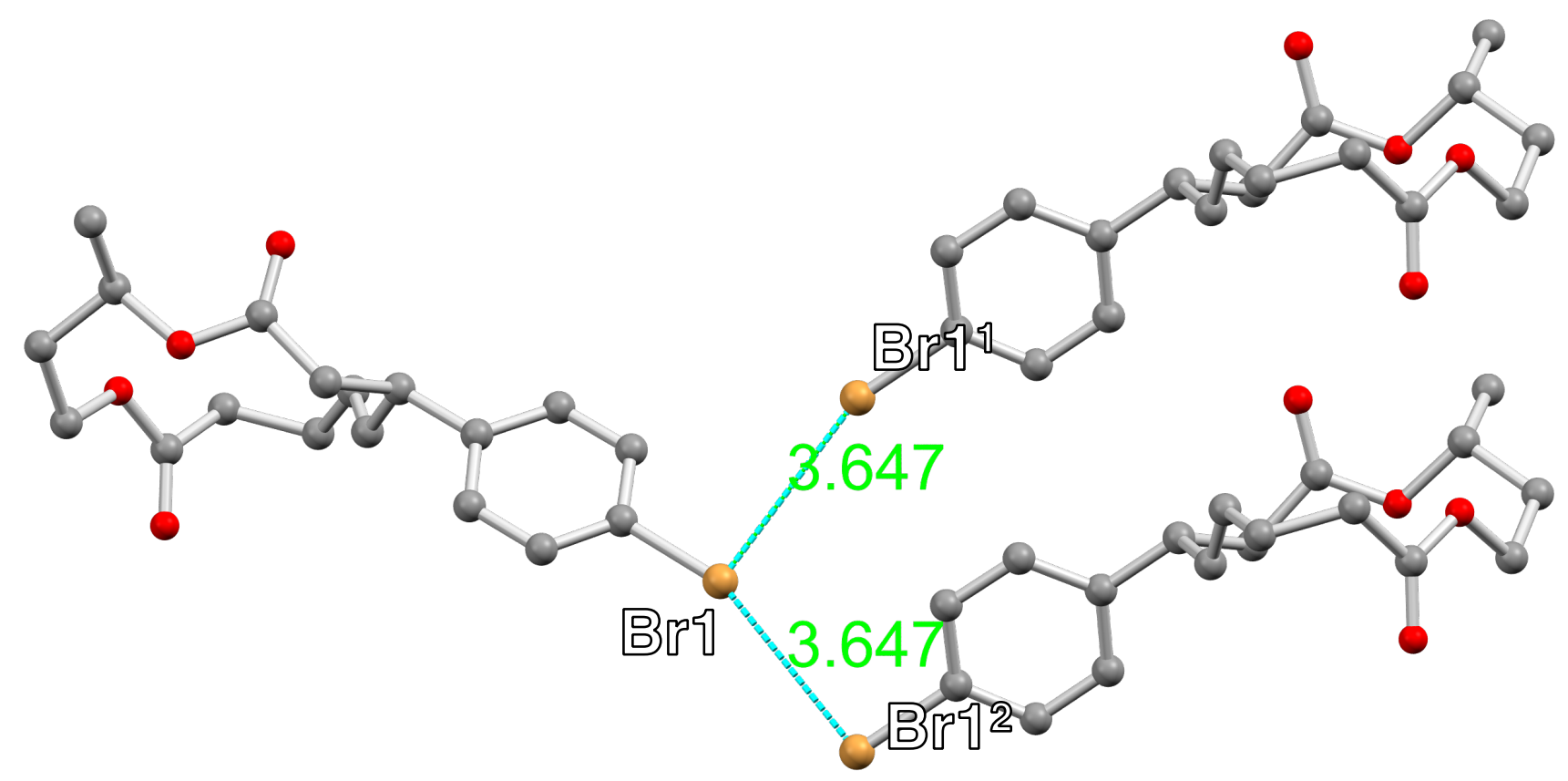

Figure S4. Interaction identified in II. Operator 1 is $\frac{3}{2}-x, \frac{1}{2}+y, \frac{1}{2}-z$; operator 2 is $\frac{3}{2}-x,-\frac{1}{2}+y, \frac{1}{2}-z$ 


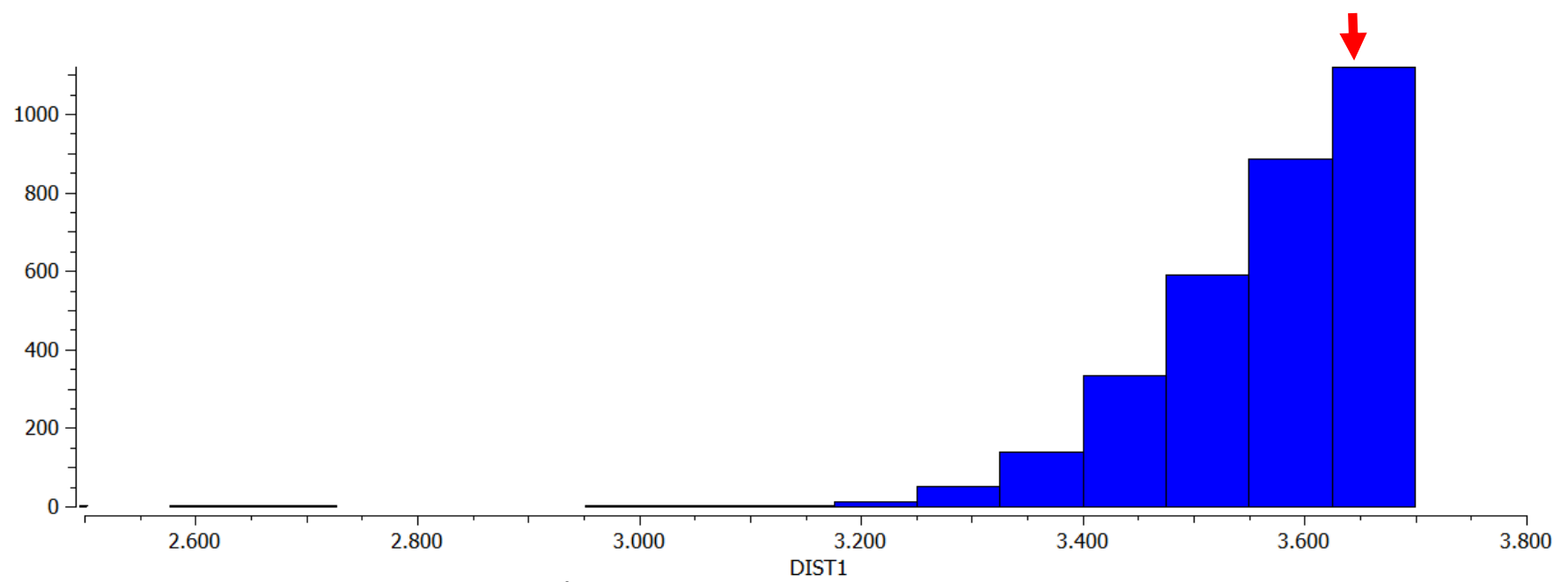

Figure S5. The distances (DIST1 in $\AA$ ) for 2486 structures when searching for fragment 2 interactions (CSD $2017+4$ updates). The red arrow identifies were the distance identified in $\mathbf{S 4}$ falls in the histogram.

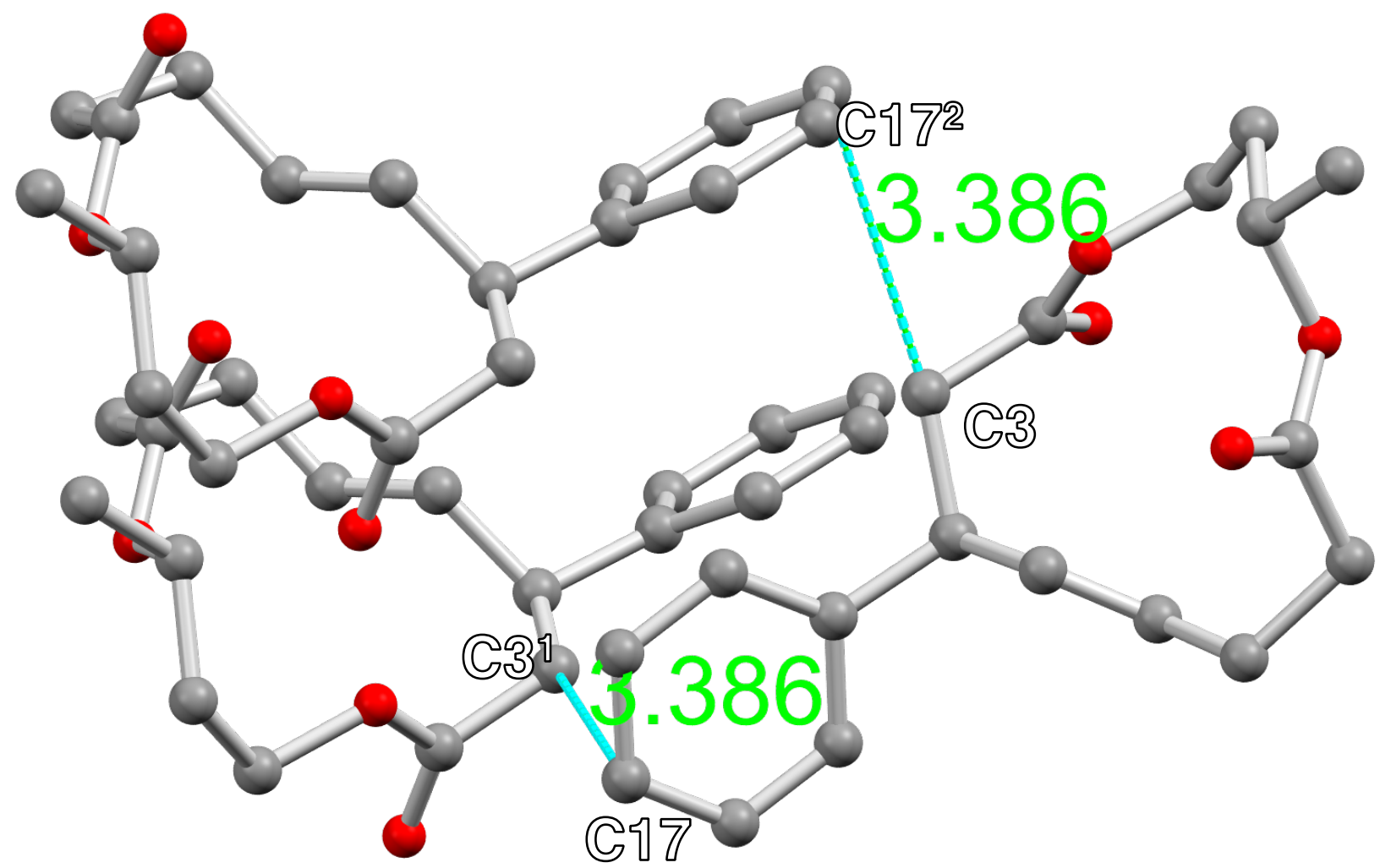

Figure S6. Interaction identified in III. Operator 1 is $1-x, 1-y,-\frac{1}{2}+z$; operator 2 is $1-x, 1-y, \frac{1}{2}+z$ 


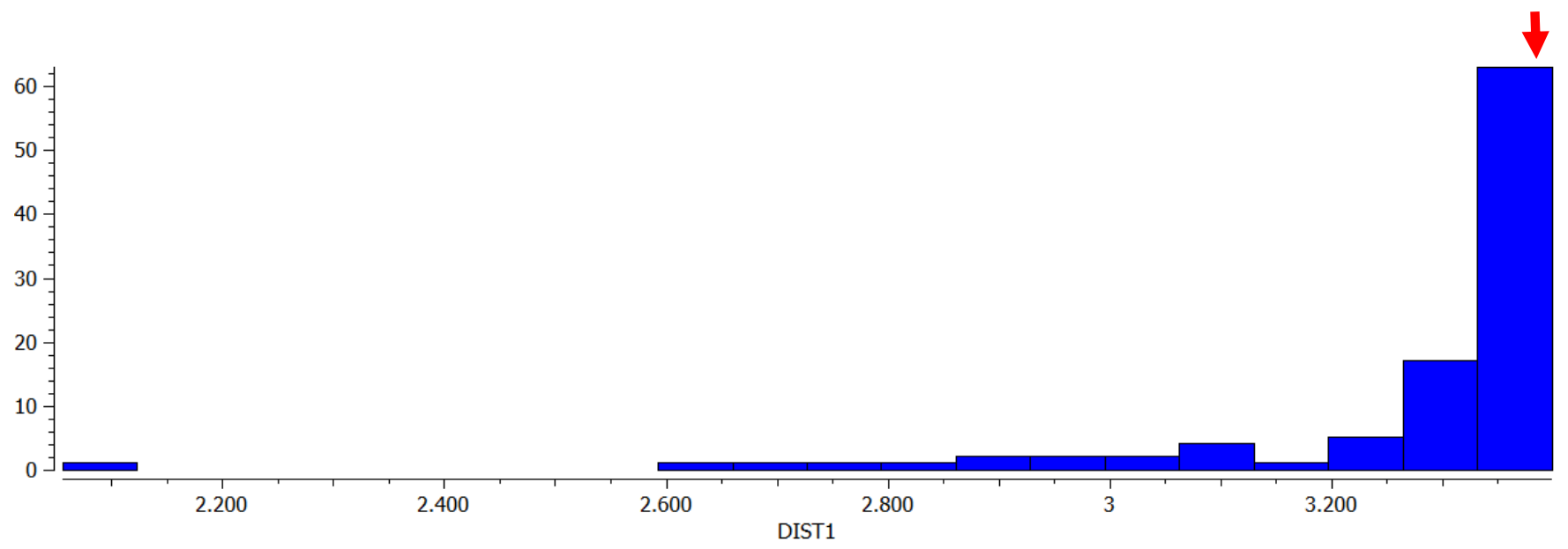

Figure S7. The distances (DIST1 in $\AA$ ) for 88 structures when searching for fragment 3 interactions (CSD $2017+4$ updates). The red arrow identifies were the distance identified in $\mathbf{S 6}$ falls in the histogram. 
Table S1. Substitution patterns and refcodes for [13]-macrodilactones

\begin{tabular}{|c|c|c|c|c|c|c|c|}
\hline Entry & Fig $X$ & Conf. & Subs.* & cis/trans & Code & Citation & Comments \\
\hline 1 & $a$ & ribbon & 11,13 & trans & URILEO & Ma \& Peczuh, 2013 & \\
\hline 3 & c & ribbon & 3 (mono) & - & XUFKOA & Magpusao, Rutledge et al., 2015 & $Z^{\prime}=2$ \\
\hline 5 & $d$ & ribbon & 3,8 & trans & XUFLAN & Magpusao, Rutledge et al., 2016 & \\
\hline 6 & $\mathrm{e}$ & ribbon & - & - & IJEHAI & Magpusao, Rutledge et al., 2016 & \\
\hline 7 & $f$ & ribbon & 3,11 & cis & IJEHOW & Magpusao, Rutledge et al., 2016 & \\
\hline 10 & $\mathrm{i}$ & ribbon & 4 (mono) & - & ECOYED & Rutledge, Hamlin et al., 2017 & \\
\hline 11 & j & ribbon & 3,13 & trans & IJEHEM & Magpusao, Rutledge et al., 2016 & \\
\hline $10^{\prime}$ & - & other & 4 (mono) & - & ECOYED & & \\
\hline 12 & - & other & 11,13 & cis & URILAK & Ma \& Peczuh, 2013 & \\
\hline $3^{\prime}$ & $c^{\prime}$ & other & 3 (mono) & - & XUFKOA & & $Z^{\prime}=2$ \\
\hline 13 & - & other & 11,13 & cis & URILAK & Ma \& Peczuh, 2013 & \\
\hline
\end{tabular}

* Subs. = positions on the [13]-macrodilactone that are substituted.

Fyvie, W.S. \& Peczuh, M.W. (2008a). Chem. Commun. 4028-4030.

Fyvie, W.S. \& Peczuh, M.W. (2008b). J. Org. Chem. 73, 3626-3629.

Ma, J. \& Peczuh, M. W. (2013). J. Org. Chem. 78, 7414-7422.

Magpusao, A.N., Rutledge, K., Mercado, B., \& Peczuh, M.W. (2015) Org. Biomol. Chem. 13, 5086-5089.

Magpusao, A.N., Rutledge, K.M., Hamlin, T.A., Lawrence, J.-M., Mercado, B.Q., Leadbeater, N.E. \& Peczuh, M.W. (2016). Chem. Eur. J. 22, 6001-6011.

Rutledge, K.M., Hamlin, T.A., Baldisseri, D., \& Peczuh, M.W. (2017). Chem. Asian J. 12, 2623-2633. 
Table S2. Aspect ratios of compounds in Figure 3 from X-ray crystallographic data

\begin{tabular}{|l|c|l|l|c|}
\hline code & cmpd & length & width & aspect ratio \\
\hline URILEO & $a$ & $5.3989(12)$ & $4.0809(13)$ & 1.323 \\
\hline KOHLAV & $b$ & $5.394(3)$ & $4.073(4)$ & 1.32 \\
\hline XUFKOA & $c$ & $5.3893(18)$ & $4.108(2)$ & 1.31 \\
\hline XOCWIW & - & $5.387(3)$ & $4.102(3)$ & 1.31 \\
\hline XUFLAN & $d$ & $5.370(4)$ & $4.098(3)$ & 1.31 \\
\hline IJEHAI & $e$ & $5.3663(11)$ & $4.1487(13)$ & 1.293 \\
\hline IJEHOW & $f$ & $5.3621(15)$ & $4.1684(16)$ & 1.286 \\
\hline II & $g$ & $5.327(2)$ & $4.272(3)$ & 1.25 \\
\hline I & $h$ & $5.327(3)$ & $4.231(4)$ & 1.26 \\
\hline ECOYED & $i$ & $5.282(5)$ & $4.383(4)$ & 1.21 \\
\hline IJEHEM & $j$ & $5.2478(18)$ & $4.365(2)$ & 1.20 \\
\hline ECOYED & - & $5.247(5)$ & $4.383(4)$ & \\
\hline URILAK & - & $5.233(4)$ & $4.391(4)$ & 1.20 \\
\hline XUFKOA & $c$ & $5.230(2)$ & $4.3762(19)$ & \\
\hline URILAX & - & $5.207(9)$ & $4.391(4)$ & \\
\hline IJEHUC & - & $5.192(2)$ & $4.245(18)$ & \\
\hline III & $k$ & $5.151(3)$ & $4.453(4)$ & \\
\hline XUFKUG & - & $4.780(2)$ & $4.4378(15)$ & \\
\hline IJEHIQ & - & $4.682(1)$ & $4.5502(15)$ & \\
\hline NOtes: & & & &
\end{tabular}

Notes:

length $=\mathrm{C} 12$ to alkene centroid distance $(\AA)$

width = C2 to C9 (carbonyl carbons) distance $(\AA)$

aspect ratio $=$ length $/$ width 
Table S3. RMSD $(\AA ̊)$ values comparing X-ray and DFT optimized structures for the core ring atoms of the [13]-macrodilactones

\begin{tabular}{lccccc}
\hline \multicolumn{1}{c}{ Compound } & \multicolumn{5}{c}{ Functional/Basis Set } \\
& $\begin{array}{c}\text { B3LYD-D3/ } \\
\text { CC-PVDC }\end{array}$ & $\begin{array}{c}\text { M06-2X-D3/ } \\
\text { CC-PVDC }\end{array}$ & $\begin{array}{c}\text { B3LYD-D3/ } \\
\text { LACVP } P^{* *}\end{array}$ & $\begin{array}{c}\text { M06-2X-D3/ } \\
\text { LACVP }\end{array}$ & average \\
\hline a (URILEO) C2/c & 0.048 & 0.070 & 0.046 & 0.057 & 0.055 \\
$e\left(\right.$ IJEHAI) $P 2_{1} / c$ & 0.061 & 0.076 & 0.113 & 0.102 & 0.088 \\
$g\left(\right.$ II) $P 2_{1} / c$ & 0.122 & 0.202 & 0.194 & 0.120 & 0.160 \\
$h\left(\right.$ I) $P 2_{1} / n$ & 0.103 & 0.119 & 0.096 & 0.094 & 0.103 \\
$k$ (III) $P$ Pna2 & 0.288 & 0.251 & 0.265 & 0.200 & 0.251 \\
\hline
\end{tabular}


Table S4. Torsion angles, bond angles, and bond lengths of compound "a" ring atoms.

\begin{tabular}{|c|c|c|c|c|c|}
\hline \multicolumn{6}{|l|}{ Torsion Angles } \\
\hline & A & B & $\mathrm{C}$ & D & XRD \\
\hline$C(2)-O(1)-C(13)-C(12)$ & 149.75 & 149.07 & 151.12 & 150.91 & 151.42 \\
\hline$O(1)-C(13)-C(12)-C(11)$ & 51.01 & 50.75 & 51.05 & 50.58 & 49.97 \\
\hline$C(13)-C(12)-C(11)-O(10)$ & 51.08 & 50.75 & 51.06 & 50.51 & 49.89 \\
\hline$C(12)-C(11)-O(10)-C(9)$ & 149.57 & 149.08 & 151.06 & 150.94 & 151.35 \\
\hline$C(11)-O(10)-C(9)-C(8)$ & 174.67 & 176.14 & 175.11 & 176.84 & 176.59 \\
\hline$O(10)-C(9)-C(8)-C(7)$ & 139.79 & 142.18 & 137.63 & 139.33 & 135.32 \\
\hline$C(9)-C(8)-C(7)-C(6)$ & 68.24 & 68.10 & 66.52 & 66.62 & 67.06 \\
\hline$C(8)-C(7)-C(6)-C(5)$ & 102.10 & 102.12 & 101.53 & 102.09 & 103.24 \\
\hline$C(7)-C(6)-C(5)-C(4)$ & 171.50 & 170.91 & 173.90 & 173.68 & 175.27 \\
\hline$C(6)-C(5)-C(4)-C(3)$ & 102.04 & 102.08 & 101.75 & 102.13 & 103.21 \\
\hline$C(5)-C(4)-C(3)-C(2)$ & 68.35 & 68.06 & 66.49 & 66.49 & 67.05 \\
\hline$C(4)-C(3)-C(2)-O(1)$ & 139.67 & 142.17 & 137.50 & 139.30 & 135.30 \\
\hline$C(3)-C(2)-O(1)-C(13)$ & 174.65 & 176.17 & 175.12 & 176.90 & 176.58 \\
\hline
\end{tabular}

\begin{tabular}{|c|c|c|c|c|c|}
\hline \multicolumn{6}{|l|}{ Bond Angles } \\
\hline & A & B & C & D & XRD \\
\hline$C(2)-O(1)-C(13)$ & 116.51 & 116.44 & 116.56 & 116.39 & 116.04 \\
\hline$O(1)-C(13)-C(12)$ & 106.62 & 106.42 & 106.40 & 106.24 & 107.16 \\
\hline$C(13)-C(12)-C(11)$ & 114.03 & 112.87 & 113.55 & 112.56 & 114.90 \\
\hline$C(12)-C(11)-O(10)$ & 106.62 & 106.41 & 106.41 & 106.23 & 107.24 \\
\hline$C(11)-O(10)-C(9)$ & 116.51 & 116.44 & 116.55 & 116.39 & 116.05 \\
\hline $\mathrm{O}(10)-\mathrm{C}(9)-\mathrm{C}(8)$ & 112.31 & 112.42 & 111.89 & 112.08 & 111.96 \\
\hline$C(9)-C(8)-C(7)$ & 110.57 & 110.12 & 110.20 & 109.72 & 110.48 \\
\hline$C(8)-C(7)-C(6)$ & 111.09 & 110.34 & 111.40 & 110.61 & 111.20 \\
\hline$C(7)-C(6)-C(5)$ & 124.92 & 124.52 & 124.45 & 124.06 & 124.73 \\
\hline$C(6)-C(5)-C(4)$ & 124.93 & 124.51 & 124.45 & 124.05 & 124.75 \\
\hline$C(5)-C(4)-C(3)$ & 111.10 & 110.34 & 111.41 & 110.62 & 111.18 \\
\hline$C(4)-C(3)-C(2)$ & 110.57 & 110.14 & 110.20 & 109.73 & 110.53 \\
\hline$C(3)-C(2)-O(1)$ & 112.31 & 112.43 & 111.90 & 112.08 & 111.99 \\
\hline
\end{tabular}

\section{Bond Lengths $(\AA)$}

\begin{tabular}{|l|r|r|r|r|r|}
\hline & \multicolumn{1}{|l|}{ A } & \multicolumn{1}{|l|}{ B } & \multicolumn{1}{l|}{ C } & \multicolumn{1}{l|}{ D } & \multicolumn{1}{l|}{ XRD } \\
\hline $\mathrm{O}(1)-\mathrm{C}(13)$ & 1.46 & 1.44 & 1.46 & 1.44 & 1.47 \\
\hline $\mathrm{C}(13)-\mathrm{C}(12)$ & 1.52 & 1.52 & 1.53 & 1.52 & 1.52 \\
\hline $\mathrm{C}(12)-\mathrm{C}(11)$ & 1.52 & 1.52 & 1.53 & 1.52 & 1.52 \\
\hline $\mathrm{C}(11)-\mathrm{O}(10)$ & 1.46 & 1.44 & 1.46 & 1.44 & 1.47 \\
\hline $\mathrm{O}(10)-\mathrm{C}(9)$ & 1.35 & 1.34 & 1.35 & 1.34 & 1.35 \\
\hline $\mathrm{C}(9)-\mathrm{C}(8)$ & 1.51 & 1.50 & 1.52 & 1.51 & 1.51 \\
\hline $\mathrm{C}(8)-\mathrm{C}(7)$ & 1.54 & 1.53 & 1.54 & 1.54 & 1.53 \\
\hline $\mathrm{C}(7)-\mathrm{C}(6)$ & 1.50 & 1.50 & 1.51 & 1.50 & 1.50 \\
\hline $\mathrm{C}(6)-\mathrm{C}(5)$ & 1.33 & 1.33 & 1.34 & 1.33 & 1.33 \\
\hline $\mathrm{C}(5)-\mathrm{C}(4)$ & 1.50 & 1.50 & 1.51 & 1.50 & 1.50 \\
\hline $\mathrm{C}(4)-\mathrm{C}(3)$ & 1.54 & 1.53 & 1.54 & 1.54 & 1.53 \\
\hline
\end{tabular}




\begin{tabular}{|l|l|l|l|l|l|}
\hline $\mathrm{C}(3)-\mathrm{C}(2)$ & 1.51 & 1.50 & 1.52 & 1.51 & 1.51 \\
\hline $\mathrm{C}(2)-\mathrm{O}(1)$ & 1.35 & 1.34 & 1.35 & 1.34 & 1.35 \\
\hline
\end{tabular}

$A=B 3 L Y P-D 3 / c c-P V D C$

$B=M 06-2 X-D 3 / c c-P V D C$

$\mathrm{C}=\mathrm{B} 3 \mathrm{LYP}-\mathrm{D} 3 / \mathrm{LACVP}{ }^{* *}$

$\mathrm{D}=\mathrm{M} 06-2 \mathrm{X}-\mathrm{D} 3 / \mathrm{LACVP}^{* *}$

Table S5. Torsion angles, bond angles, and bond lengths of compound " $g$ " (II) ring atoms.

\begin{tabular}{|c|c|c|c|c|c|}
\hline & A & B & C & D & XRD \\
\hline$C(2)-O(1)-C(13)-C(12)$ & 149.63 & 149.90 & 150.79 & 151.03 & 155.42 \\
\hline $\mathrm{O}(1)-\mathrm{C}(13)-\mathrm{C}(12)-\mathrm{C}(11)$ & 49.61 & 49.76 & 50.33 & 49.90 & 50.10 \\
\hline$C(13)-C(12)-C(11)-O(10)$ & 52.09 & 50.87 & 52.55 & 51.98 & 49.63 \\
\hline $\mathrm{C}(12)-\mathrm{C}(11)-\mathrm{O}(10)-\mathrm{C}(9)$ & 148.03 & 142.56 & 150.06 & 149.59 & 161.76 \\
\hline$C(11)-O(10)-C(9)-C(8)$ & 174.74 & 175.80 & 174.07 & 176.19 & 174.91 \\
\hline$O(10)-C(9)-C(8)-C(7)$ & 141.28 & 148.04 & 138.64 & 140.44 & 125.30 \\
\hline$C(9)-C(8)-C(7)-C(6)$ & 67.97 & 67.99 & 65.61 & 65.74 & 70.30 \\
\hline$C(8)-C(7)-C(6)-C(5)$ & 102.03 & 102.05 & 99.99 & 101.74 & 105.86 \\
\hline$C(7)-C(6)-C(5)-C(4)$ & 171.51 & 169.43 & 175.23 & 174.15 & 177.36 \\
\hline$C(6)-C(5)-C(4)-C(3)$ & 103.55 & 102.12 & 102.41 & 103.05 & 107.00 \\
\hline$C(5)-C(4)-C(3)-C(2)$ & 70.28 & 68.88 & 65.58 & 68.14 & 71.58 \\
\hline$C(4)-C(3)-C(2)-O(1)$ & 141.37 & 144.57 & 137.99 & 140.69 & 132.10 \\
\hline$C(3)-C(2)-O(1)-C(13)$ & 175.88 & 179.16 & 175.78 & 176.63 & 174.11 \\
\hline
\end{tabular}

\begin{tabular}{|c|c|c|c|c|c|}
\hline \multicolumn{6}{|l|}{ Bond Angles } \\
\hline & A & B & C & D & XRD \\
\hline$C(2)-O(1)-C(13)$ & 116.42 & 116.17 & 116.80 & 116.50 & 116.30 \\
\hline$O(1)-C(13)-C(12)$ & 106.78 & 106.79 & 106.31 & 106.18 & 105.89 \\
\hline$C(13)-C(12)-C(11)$ & 113.43 & 112.41 & 112.90 & 111.94 & 113.93 \\
\hline$C(12)-C(11)-O(10)$ & 108.36 & 108.12 & 107.81 & 107.53 & 107.42 \\
\hline$C(11)-O(10)-C(9)$ & 116.54 & 116.73 & 116.73 & 116.50 & 115.77 \\
\hline $\mathrm{O}(10)-\mathrm{C}(9)-\mathrm{C}(8)$ & 112.10 & 112.04 & 111.60 & 111.90 & 111.61 \\
\hline$C(9)-C(8)-C(7)$ & 110.59 & 110.44 & 110.15 & 109.74 & 110.55 \\
\hline$C(8)-C(7)-C(6)$ & 110.96 & 110.06 & 111.47 & 110.49 & 111.75 \\
\hline$C(7)-C(6)-C(5)$ & 124.96 & 124.63 & 124.03 & 123.80 & 124.21 \\
\hline$C(6)-C(5)-C(4)$ & 124.88 & 124.27 & 124.66 & 124.20 & 124.33 \\
\hline$C(5)-C(4)-C(3)$ & 109.20 & 108.38 & 110.25 & 109.33 & 109.79 \\
\hline$C(4)-C(3)-C(2)$ & 110.46 & 110.17 & 110.42 & 109.43 & 109.71 \\
\hline$C(3)-C(2)-O(1)$ & 112.29 & 112.54 & 111.56 & 111.77 & 112.20 \\
\hline \multicolumn{6}{|l|}{ Bond Lengths (Å) } \\
\hline & A & B & C & $\mathrm{D}$ & XRD \\
\hline $\mathrm{O}(1)-\mathrm{C}(13)$ & 1.46 & 1.45 & 1.46 & 1.44 & 1.47 \\
\hline$C(13)-C(12)$ & 1.52 & 1.52 & 1.53 & 1.52 & 1.52 \\
\hline$C(12)-C(11)$ & 1.51 & 1.51 & 1.52 & 1.52 & 1.51 \\
\hline$C(11)-O(10)$ & 1.45 & 1.43 & 1.45 & 1.43 & 1.45 \\
\hline
\end{tabular}




\begin{tabular}{|l|l|l|l|l|l|}
\hline $\mathrm{O}(10)-\mathrm{C}(9)$ & 1.35 & 1.34 & 1.35 & 1.34 & 1.34 \\
\hline $\mathrm{C}(9)-\mathrm{C}(8)$ & 1.51 & 1.50 & 1.52 & 1.51 & 1.51 \\
\hline $\mathrm{C}(8)-\mathrm{C}(7)$ & 1.53 & 1.53 & 1.54 & 1.54 & 1.54 \\
\hline $\mathrm{C}(7)-\mathrm{C}(6)$ & 1.50 & 1.50 & 1.51 & 1.50 & 1.50 \\
\hline $\mathrm{C}(6)-\mathrm{C}(5)$ & 1.33 & 1.33 & 1.34 & 1.33 & 1.33 \\
\hline $\mathrm{C}(5)-\mathrm{C}(4)$ & 1.51 & 1.50 & 1.51 & 1.51 & 1.51 \\
\hline $\mathrm{C}(4)-\mathrm{C}(3)$ & 1.54 & 1.53 & 1.55 & 1.54 & 1.55 \\
\hline $\mathrm{C}(3)-\mathrm{C}(2)$ & 1.51 & 1.50 & 1.52 & 1.51 & 1.51 \\
\hline $\mathrm{C}(2)-\mathrm{O}(1)$ & 1.35 & 1.34 & 1.35 & 1.34 & 1.34 \\
\hline
\end{tabular}

$\mathrm{A}=\mathrm{B} 3 \mathrm{LYP}-\mathrm{D} 3 / \mathrm{cc}-\mathrm{PVDC}$

$B=M 06-2 X-D 3 / c c-P V D C$

$\mathrm{C}=\mathrm{B} 3 \mathrm{LYP}-\mathrm{D} 3 / \mathrm{LACVP}^{* *}$

$D=M 06-2 X-D 3 / L A C V P^{* *}$

Table S6. Torsion angles, bond angles, and bond lengths of compound "e" ring atoms.

Torsion Angles

\begin{tabular}{|l|r|r|r|r|r|}
\hline & \multicolumn{1}{l|}{ A } & \multicolumn{1}{l|}{ C } & \multicolumn{1}{l|}{ D } & \multicolumn{1}{l|}{ XRD } \\
\hline$C(2)-O(1)-C(13)-C(12)$ & 156.67 & 158.96 & 153.30 & 151.10 & 168.10 \\
\hline$O(1)-C(13)-C(12)-C(11)$ & 51.95 & 51.86 & 52.14 & 51.53 & 52.76 \\
\hline$C(13)-C(12)-C(11)-O(10)$ & 51.56 & 50.79 & 51.85 & 51.45 & 48.62 \\
\hline$C(12)-C(11)-O(10)-C(9)$ & 145.37 & 139.08 & 149.48 & 149.56 & 141.96 \\
\hline$C(11)-O(10)-C(9)-C(8)$ & 173.78 & 174.55 & 173.94 & 175.96 & 172.56 \\
\hline$O(10)-C(9)-C(8)-C(7)$ & 140.99 & 147.55 & 137.94 & 140.34 & 142.12 \\
\hline$C(9)-C(8)-C(7)-C(6)$ & 67.65 & 67.05 & 66.53 & 66.40 & 67.65 \\
\hline$C(8)-C(7)-C(6)-C(5)$ & 102.24 & 102.43 & 101.92 & 102.16 & 101.74 \\
\hline$C(7)-C(6)-C(5)-C(4)$ & 172.93 & 171.51 & 174.47 & 173.73 & 175.46 \\
\hline$C(6)-C(5)-C(4)-C(3)$ & 103.77 & 102.69 & 102.09 & 102.09 & 103.93 \\
\hline$C(5)-C(4)-C(3)-C(2)$ & 69.16 & 68.81 & 66.84 & 66.60 & 66.45 \\
\hline$C(4)-C(3)-C(2)-O(1)$ & 133.64 & 135.48 & 135.67 & 139.46 & 122.45 \\
\hline$C(3)-C(2)-O(1)-C(13)$ & 174.30 & 176.82 & 174.31 & 176.11 & 176.69 \\
\hline$B$ Bond $A n g$ & & & & &
\end{tabular}

Bond Angles

\begin{tabular}{|l|l|l|l|l|l|}
\hline & $A$ & $B$ & $C$ & $D$ & XRD \\
\hline$C(2)-O(1)-C(13)$ & 116.08 & 115.72 & 116.38 & 116.35 & 115.65 \\
\hline$O(1)-C(13)-C(12)$ & 108.10 & 107.88 & 107.80 & 107.54 & 107.44 \\
\hline$C(13)-C(12)-C(11)$ & 112.71 & 111.78 & 112.29 & 111.26 & 112.90 \\
\hline$C(12)-C(11)-O(10)$ & 108.22 & 108.08 & 107.81 & 107.55 & 107.17 \\
\hline$C(11)-O(10)-C(9)$ & 116.70 & 116.90 & 116.65 & 116.46 & 117.04 \\
\hline$O(10)-C(9)-C(8)$ & 111.99 & 111.90 & 111.67 & 111.90 & 111.39 \\
\hline$C(9)-C(8)-C(7)$ & 110.55 & 110.41 & 110.24 & 109.74 & 110.59 \\
\hline$C(8)-C(7)-C(6)$ & 111.43 & 110.71 & 111.62 & 110.64 & 111.20 \\
\hline$C(7)-C(6)-C(5)$ & 124.75 & 124.18 & 124.37 & 124.01 & 125.29 \\
\hline$C(6)-C(5)-C(4)$ & 125.18 & 124.87 & 124.52 & 124.07 & 124.52 \\
\hline$C(5)-C(4)-C(3)$ & 111.30 & 110.19 & 111.50 & 110.60 & 112.54 \\
\hline$C(4)-C(3)-C(2)$ & 110.22 & 109.63 & 110.11 & 109.66 & 110.40 \\
\hline
\end{tabular}




\begin{tabular}{|c|c|c|c|c|c|}
\hline$C(3)-C(2)-O(1)$ & 112.44 & 112.72 & 111.83 & 111.97 & 112.40 \\
\hline \multicolumn{6}{|c|}{ Bond Lengths ( $(\AA)$} \\
\hline & A & B & C & $\mathrm{D}$ & XRD \\
\hline $\mathrm{O}(1)-\mathrm{C}(13)$ & 1.44 & 1.43 & 1.45 & 1.43 & 1.46 \\
\hline$C(13)-C(12)$ & 1.52 & 1.51 & 1.52 & 1.52 & 1.52 \\
\hline$C(12)-C(11)$ & 1.52 & 1.51 & 1.52 & 1.52 & 1.52 \\
\hline$C(11)-O(10)$ & 1.45 & 1.43 & 1.45 & 1.43 & 1.45 \\
\hline $\mathrm{O}(10)-\mathrm{C}(9)$ & 1.35 & 1.35 & 1.35 & 1.34 & 1.35 \\
\hline$C(9)-C(8)$ & 1.51 & 1.51 & 1.52 & 1.51 & 1.51 \\
\hline$C(8)-C(7)$ & 1.54 & 1.53 & 1.54 & 1.54 & 1.54 \\
\hline$C(7)-C(6)$ & 1.50 & 1.50 & 1.51 & 1.50 & 1.50 \\
\hline$C(6)-C(5)$ & 1.33 & 1.33 & 1.34 & 1.33 & 1.32 \\
\hline$C(5)-C(4)$ & 1.50 & 1.50 & 1.51 & 1.50 & 1.51 \\
\hline$C(4)-C(3)$ & 1.54 & 1.53 & 1.55 & 1.54 & 1.54 \\
\hline$C(3)-C(2)$ & 1.51 & 1.50 & 1.52 & 1.51 & 1.51 \\
\hline$C(2)-O(1)$ & 1.35 & 1.34 & 1.35 & 1.34 & 1.35 \\
\hline
\end{tabular}

$\mathrm{A}=\mathrm{B} 3 \mathrm{LYP}-\mathrm{D} 3 / \mathrm{cc}-\mathrm{PVDC}$

$B=M 06-2 X-D 3 / c C-P V D C$

$\mathrm{C}=\mathrm{B} 3 \mathrm{LYP}-\mathrm{D} 3 / \mathrm{LACVP}{ }^{* *}$

$D=M 06-2 X-D 3 / L A C V P^{* *}$

Table S7. Torsion angles, bond angles, and bond lengths of compound " $h$ " I ring atoms.

\begin{tabular}{|c|c|c|c|c|c|}
\hline \multicolumn{6}{|l|}{ Torsion Angles } \\
\hline & A & B & C & D & XRD \\
\hline$C(2)-O(1)-C(13)-C(12)$ & 150.99 & 148.95 & 149.91 & 148.44 & 151.07 \\
\hline $\mathrm{O}(1)-\mathrm{C}(13)-\mathrm{C}(12)-\mathrm{C}(11)$ & 51.49 & 51.12 & 51.70 & 51.20 & 49.64 \\
\hline$C(13)-C(12)-C(11)-O(10)$ & 51.52 & 51.22 & 51.95 & 51.66 & 50.46 \\
\hline$C(12)-C(11)-O(10)-C(9)$ & 149.95 & 149.11 & 153.66 & 155.57 & 166.35 \\
\hline$C(11)-O(10)-C(9)-C(8)$ & 174.65 & 176.37 & 174.62 & 176.45 & 173.06 \\
\hline $\mathrm{O}(10)-\mathrm{C}(9)-\mathrm{C}(8)-\mathrm{C}(7)$ & 138.59 & 141.69 & 135.27 & 135.44 & 125.79 \\
\hline$C(9)-C(8)-C(7)-C(6)$ & 66.88 & 66.58 & 65.94 & 65.73 & 73.62 \\
\hline$C(8)-C(7)-C(6)-C(5)$ & 102.68 & 102.31 & 102.60 & 102.64 & 101.60 \\
\hline$C(7)-C(6)-C(5)-C(4)$ & 175.47 & 174.06 & 177.43 & 177.34 & 177.42 \\
\hline$C(6)-C(5)-C(4)-C(3)$ & 102.17 & 101.85 & 101.81 & 102.37 & 109.64 \\
\hline$C(5)-C(4)-C(3)-C(2)$ & 67.23 & 66.79 & 65.80 & 65.50 & 66.32 \\
\hline$C(4)-C(3)-C(2)-O(1)$ & 138.19 & 142.01 & 137.78 & 139.80 & 132.19 \\
\hline$C(3)-C(2)-O(1)-C(13)$ & 174.58 & 176.21 & 174.15 & 175.35 & 174.67 \\
\hline \multicolumn{6}{|l|}{ Bond Angles } \\
\hline & A & $B$ & $\mathrm{C}$ & $\mathrm{D}$ & XRD \\
\hline$C(2)-O(1)-C(13)$ & 116.45 & 116.42 & 116.60 & 116.55 & 116.52 \\
\hline$O(1)-C(13)-C(12)$ & 108.09 & 107.86 & 107.76 & 107.47 & 107.50 \\
\hline$C(13)-C(12)-C(11)$ & 112.57 & 111.55 & 112.20 & 111.20 & 112.67 \\
\hline$C(12)-C(11)-O(10)$ & 108.09 & 107.85 & 107.75 & 107.44 & 107.50 \\
\hline$C(11)-O(10)-C(9)$ & 116.55 & 116.43 & 116.38 & 116.06 & 115.27 \\
\hline
\end{tabular}




\begin{tabular}{|l|l|l|l|l|l|}
\hline $\mathrm{O}(10)-\mathrm{C}(9)-\mathrm{C}(8)$ & 112.10 & 112.26 & 111.86 & 112.17 & 112.89 \\
\hline $\mathrm{C}(9)-\mathrm{C}(8)-\mathrm{C}(7)$ & 111.15 & 110.78 & 110.63 & 110.08 & 111.52 \\
\hline $\mathrm{C}(8)-\mathrm{C}(7)-\mathrm{C}(6)$ & 109.88 & 109.19 & 110.17 & 109.61 & 109.75 \\
\hline $\mathrm{C}(7)-\mathrm{C}(6)-\mathrm{C}(5)$ & 125.32 & 124.93 & 125.02 & 124.76 & 125.22 \\
\hline $\mathrm{C}(6)-\mathrm{C}(5)-\mathrm{C}(4)$ & 125.42 & 124.99 & 124.93 & 124.51 & 126.13 \\
\hline $\mathrm{C}(5)-\mathrm{C}(4)-\mathrm{C}(3)$ & 109.84 & 109.18 & 110.23 & 109.78 & 110.36 \\
\hline $\mathrm{C}(4)-\mathrm{C}(3)-\mathrm{C}(2)$ & 111.13 & 110.78 & 110.78 & 110.37 & 112.29 \\
\hline $\mathrm{C}(3)-\mathrm{C}(2)-\mathrm{O}(1)$ & 112.16 & 112.27 & 111.72 & 111.88 & 112.05 \\
\hline
\end{tabular}

\section{Bond Lengths $(\AA)$}

\begin{tabular}{|l|r|r|r|r|r|}
\hline & \multicolumn{1}{|l|}{ A } & \multicolumn{1}{l|}{ B } & \multicolumn{1}{l|}{ C } & \multicolumn{1}{l|}{ XRD } \\
\hline $\mathrm{O}(1)-\mathrm{C}(13)$ & 1.44 & 1.43 & 1.45 & 1.43 & 1.45 \\
\hline $\mathrm{C}(13)-\mathrm{C}(12)$ & 1.52 & 1.51 & 1.52 & 1.52 & 1.51 \\
\hline $\mathrm{C}(12)-\mathrm{C}(11)$ & 1.52 & 1.51 & 1.52 & 1.52 & 1.50 \\
\hline $\mathrm{C}(11)-\mathrm{O}(10)$ & 1.44 & 1.43 & 1.45 & 1.43 & 1.45 \\
\hline $\mathrm{O}(10)-\mathrm{C}(9)$ & 1.35 & 1.34 & 1.35 & 1.34 & 1.33 \\
\hline $\mathrm{C}(9)-\mathrm{C}(8)$ & 1.51 & 1.50 & 1.52 & 1.51 & 1.50 \\
\hline $\mathrm{C}(8)-\mathrm{C}(7)$ & 1.54 & 1.53 & 1.55 & 1.54 & 1.53 \\
\hline $\mathrm{C}(7)-\mathrm{C}(6)$ & 1.50 & 1.50 & 1.51 & 1.51 & 1.51 \\
\hline $\mathrm{C}(6)-\mathrm{C}(5)$ & 1.33 & 1.33 & 1.34 & 1.33 & 1.30 \\
\hline $\mathrm{C}(5)-\mathrm{C}(4)$ & 1.50 & 1.50 & 1.51 & 1.51 & 1.50 \\
\hline $\mathrm{C}(4)-\mathrm{C}(3)$ & 1.54 & 1.53 & 1.55 & 1.54 & 1.52 \\
\hline $\mathrm{C}(3)-\mathrm{C}(2)$ & 1.51 & 1.50 & 1.52 & 1.51 & 1.49 \\
\hline $\mathrm{C}(2)-\mathrm{O}(1)$ & 1.35 & 1.34 & 1.35 & 1.34 & 1.34 \\
\hline$A$
\end{tabular}

$\mathrm{A}=\mathrm{B} 3 \mathrm{LYP}-\mathrm{D} 3 / \mathrm{cc}-\mathrm{PVDC}$

$B=$ M06-2X-D3/CC-PVDC

$\mathrm{C}=\mathrm{B} 3 \mathrm{LYP}-\mathrm{D} 3 / \mathrm{LACV} \mathrm{P}^{* *}$

$\mathrm{D}=\mathrm{M} 06-2 \mathrm{X}-\mathrm{D} 3 / \mathrm{LACVP} \mathrm{P}^{* *}$

Table S8. Torsion angles, bond angles, and bond lengths of compound " $k$ " III ring atoms.

\begin{tabular}{|l|r|r|r|r|r|}
\hline Torsion Angles & \multicolumn{7}{|l|}{ A } & \multicolumn{1}{l|}{ C } & D & XRD \\
\hline$C(2)-O(1)-C(13)-C(12)$ & 146.27 & 148.07 & 149.18 & 150.05 & 156.26 \\
\hline$O(1)-C(13)-C(12)-C(11)$ & 49.66 & 49.20 & 50.75 & 50.35 & 44.67 \\
\hline$C(13)-C(12)-C(11)-O(10)$ & 52.26 & 51.79 & 52.60 & 52.06 & 51.24 \\
\hline$C(12)-C(11)-O(10)-C(9)$ & 150.26 & 147.45 & 153.50 & 153.34 & 173.45 \\
\hline$C(11)-O(10)-C(9)-C(8)$ & 175.43 & 177.30 & 174.31 & 176.11 & 176.09 \\
\hline$O(10)-C(9)-C(8)-C(7)$ & 141.07 & 144.58 & 135.55 & 136.94 & 110.82 \\
\hline$C(9)-C(8)-C(7)-C(6)$ & 67.89 & 67.44 & 65.07 & 65.49 & 68.45 \\
\hline$C(8)-C(7)-C(6)-C(5)$ & 103.12 & 103.72 & 103.53 & 104.97 & 117.02 \\
\hline$C(7)-C(6)-C(5)-C(4)$ & 171.22 & 170.71 & 175.93 & 176.14 & 177.15 \\
\hline$C(6)-C(5)-C(4)-C(3)$ & 101.02 & 101.28 & 99.77 & 100.31 & 102.70 \\
\hline$C(5)-C(4)-C(3)-C(2)$ & 68.92 & 69.36 & 66.31 & 66.64 & 72.75 \\
\hline$C(4)-C(3)-C(2)-O(1)$ & 143.01 & 143.80 & 138.50 & 139.18 & 125.06 \\
\hline$C(3)-C(2)-O(1)-C(13)$ & 175.31 & 177.06 & 174.93 & 176.38 & 175.80 \\
\hline
\end{tabular}




\begin{tabular}{|c|c|c|c|c|c|}
\hline \multicolumn{6}{|l|}{ Bond Angles } \\
\hline & $A$ & $B$ & $\mathrm{C}$ & $\mathrm{D}$ & XRD \\
\hline$C(2)-O(1)-C(13)$ & 116.57 & 116.30 & 116.61 & 116.40 & 115.95 \\
\hline $\mathrm{O}(1)-\mathrm{C}(13)-\mathrm{C}(12)$ & 106.77 & 106.63 & 106.35 & 106.24 & 106.29 \\
\hline$C(13)-C(12)-C(11)$ & 113.23 & 112.28 & 112.77 & 111.87 & 115.19 \\
\hline$C(12)-C(11)-O(10)$ & 108.24 & 108.06 & 107.66 & 107.37 & 107.63 \\
\hline$C(11)-O(10)-C(9)$ & 116.49 & 116.48 & 116.55 & 116.30 & 115.40 \\
\hline $\mathrm{O}(10)-\mathrm{C}(9)-\mathrm{C}(8)$ & 112.11 & 112.08 & 111.67 & 111.82 & 111.91 \\
\hline$C(9)-C(8)-C(7)$ & 110.74 & 110.19 & 110.34 & 109.46 & 110.36 \\
\hline$C(8)-C(7)-C(6)$ & 109.48 & 108.86 & 110.41 & 109.73 & 110.92 \\
\hline$C(7)-C(6)-C(5)$ & 124.65 & 124.20 & 124.71 & 124.24 & 123.81 \\
\hline$C(6)-C(5)-C(4)$ & 124.95 & 124.81 & 124.06 & 123.92 & 123.73 \\
\hline$C(5)-C(4)-C(3)$ & 110.86 & 109.96 & 111.57 & 110.77 & 112.41 \\
\hline$C(4)-C(3)-C(2)$ & 110.78 & 110.16 & 110.19 & 109.55 & 109.04 \\
\hline$C(3)-C(2)-O(1)$ & 112.25 & 112.55 & 111.84 & 112.07 & 112.43 \\
\hline \multicolumn{6}{|l|}{ Bond Lengths ( $\AA$ ) } \\
\hline & A & $B$ & $\mathrm{C}$ & $\mathrm{D}$ & XRD \\
\hline $\mathrm{O}(1)-\mathrm{C}(13)$ & 1.46 & 1.44 & 1.46 & 1.44 & 1.45 \\
\hline$C(13)-C(12)$ & 1.52 & 1.52 & 1.53 & 1.52 & 1.51 \\
\hline$C(12)-C(11)$ & 1.51 & 1.51 & 1.52 & 1.52 & 1.50 \\
\hline$C(11)-O(10)$ & 1.45 & 1.43 & 1.45 & 1.43 & 1.44 \\
\hline $\mathrm{O}(10)-\mathrm{C}(9)$ & 1.35 & 1.34 & 1.35 & 1.34 & 1.34 \\
\hline$C(9)-C(8)$ & 1.50 & 1.50 & 1.52 & 1.51 & 1.49 \\
\hline$C(8)-C(7)$ & 1.54 & 1.53 & 1.55 & 1.53 & 1.53 \\
\hline$C(7)-C(6)$ & 1.50 & 1.50 & 1.51 & 1.51 & 1.50 \\
\hline$C(6)-C(5)$ & 1.33 & 1.33 & 1.34 & 1.33 & 1.31 \\
\hline$C(5)-C(4)$ & 1.50 & 1.49 & 1.51 & 1.50 & 1.49 \\
\hline$C(4)-C(3)$ & 1.53 & 1.53 & 1.54 & 1.54 & 1.52 \\
\hline$C(3)-C(2)$ & 1.51 & 1.50 & 1.52 & 1.51 & 1.50 \\
\hline $\mathrm{C}(2)-\mathrm{O}(1)$ & 1.35 & 1.34 & 1.35 & 1.34 & 1.33 \\
\hline \multicolumn{6}{|c|}{$\begin{array}{l}A=B 3 L Y P-D 3 / c c-P V D C \\
B=\text { M06-2X-D3/cc-PVDC } \\
C=B 3 L Y P-D 3 / L A C V P^{* *} \\
D=M 06-2 X-D 3 / L A C V P^{* *}\end{array}$} \\
\hline
\end{tabular}



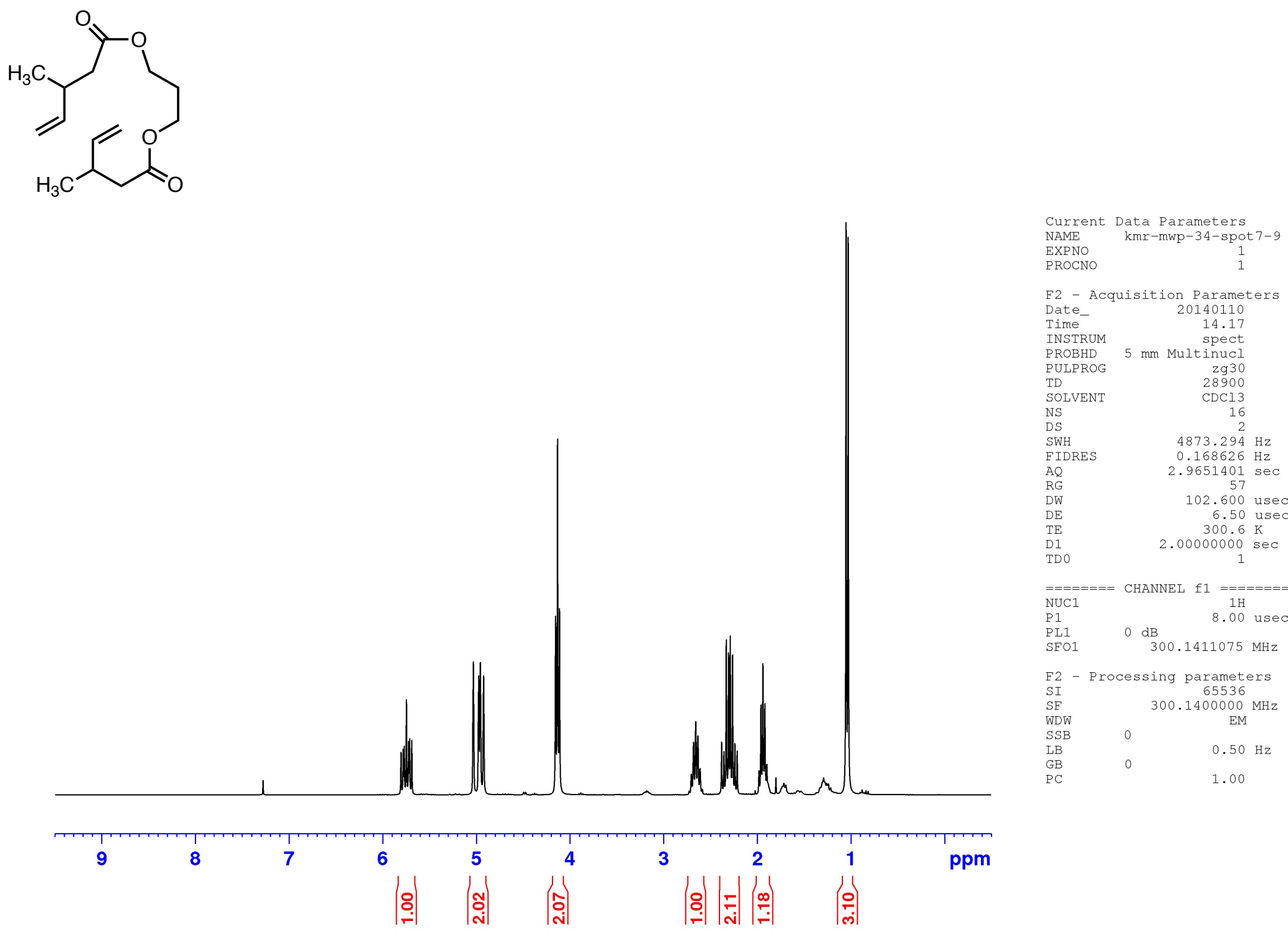

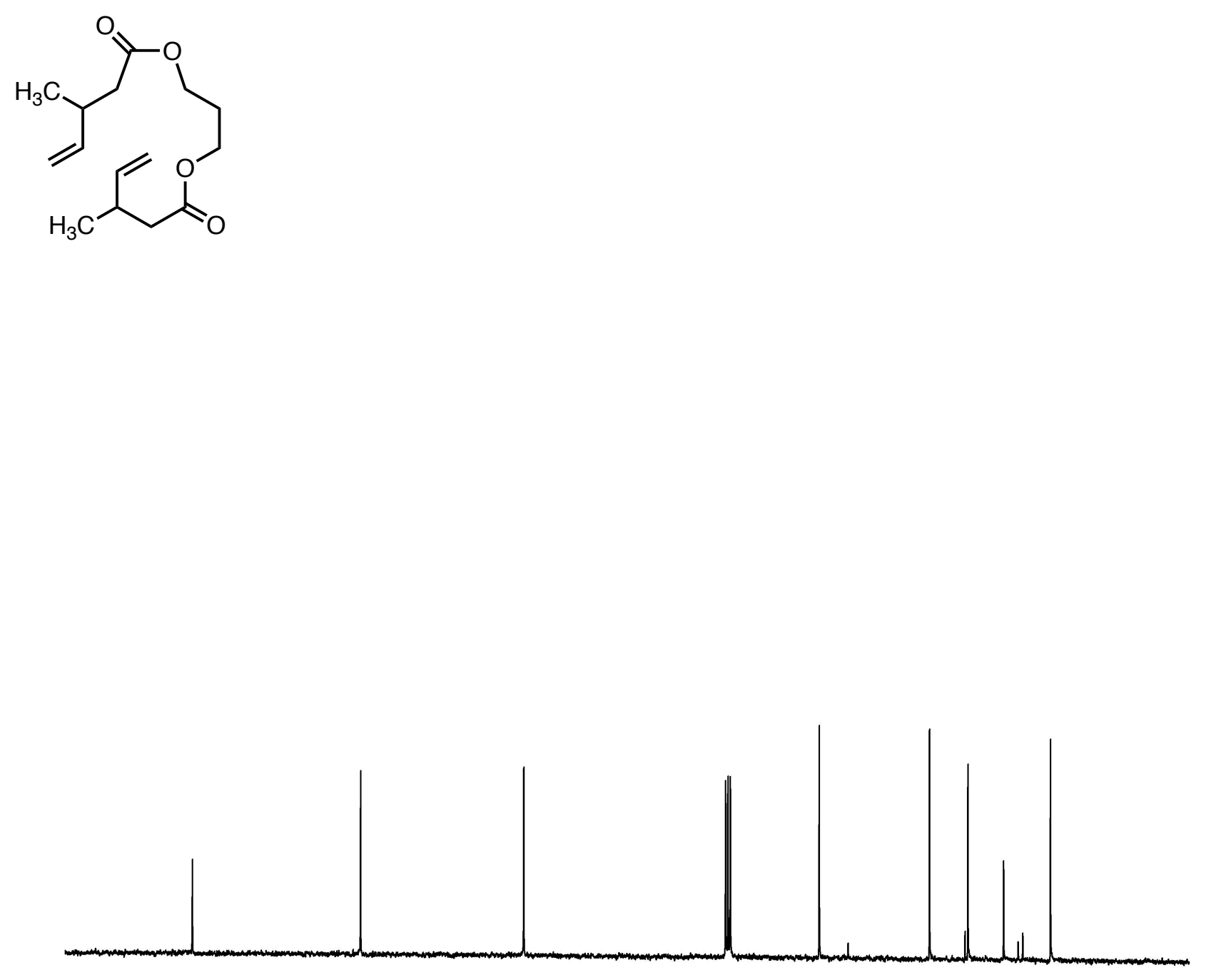

Current Data Parameters
NAME
kmr-mwp-34-spot7-9 EXPNO PROCNO

F2 - Acquisition Parameters Date $\quad 2014011$

INSTRUM spect

PROBHD $5 \mathrm{~mm}$ Multinucl

$\begin{array}{lr}\text { PULPROG } & \text { zgpg30 } \\ \text { TD } & 32696\end{array}$

SOLVENT

NS
DS

$\begin{array}{ll}4 & 4 \\ \text { SWH } & 17985.611 \mathrm{~Hz}\end{array}$

$\begin{array}{ll}\text { FIDRES } & 0.550086 \mathrm{~Hz}\end{array}$

RG $\quad 181$

$\begin{array}{lr}\mathrm{DW} & 27.800 \text { usec } \\ \mathrm{DE} & 6.50 \mathrm{usec}\end{array}$

TE $\quad 304.9 \mathrm{~K}$

$\begin{array}{ll}\text { D1 } & 1.50000000 \mathrm{sec} \\ \text { D11 } & 0.03000000 \mathrm{sec}\end{array}$

D11
TD0

$========$ CHANNEL $\mathrm{fl}========$
NUC1
$13 \mathrm{C}$

P1 $\quad 8.50$ usec

$\begin{array}{lll}\text { PL1 } & 0 \mathrm{~dB} \\ \text { SFO1 } & & 75.4775590 \mathrm{MHz}\end{array}$

$=======$ CHANNEL $\mathrm{f} 2======$

CPDPRG [2 waltz16

NUC2 $1 \mathrm{H}$

$\begin{array}{lr}\text { PCPD2 } & 100.00 \text { usec } \\ \text { PI2 } & -5.00 \mathrm{~dB}\end{array}$

PL2
PL12

$8.56 \mathrm{~dB}$

$20.00 \mathrm{~dB}$

$\mathrm{SHO} 2 \mathrm{M}-1402005 \mathrm{MHz}$

F2 - Processing parameters

SI $\quad 731072 \mathrm{MH}$

$\begin{array}{lr}\text { WDW } & 75.4702630 \\ \text { SSB } & \text { EM }\end{array}$

$\begin{array}{lll}\mathrm{SSB} & 0 \\ \mathrm{LB} & 2.50 \mathrm{~Hz}\end{array}$

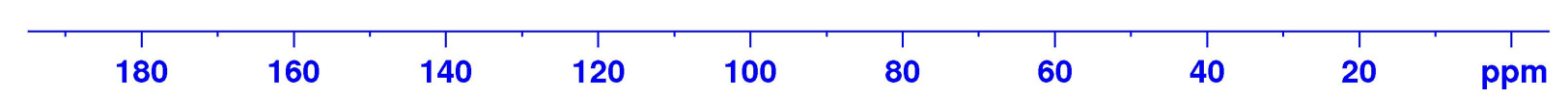

$\begin{array}{ll}\mathrm{GB} & 0 \\ \mathrm{PC} & \end{array}$ 

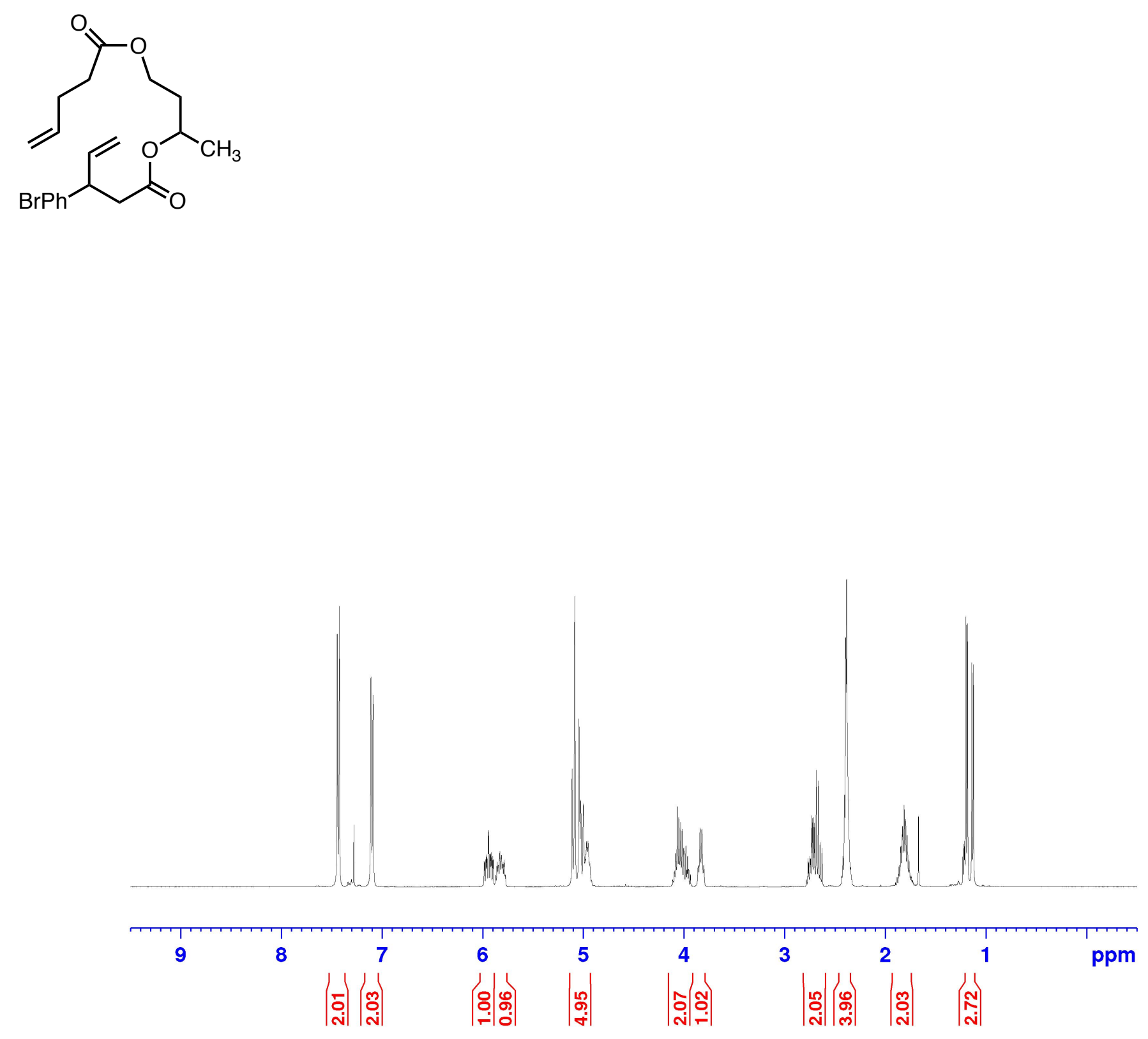

Current Data Parameters

NAME kmr-mwp-43-c9

EXPNO

F2 - Acquisition Parameters

$\begin{array}{lr}\text { Date } & 20140916 \\ \text { Time- } & 15.27\end{array}$

INSTRUM spect

PROBHD $5 \mathrm{~mm}$ PABBO BB/

PULPROG $\quad$ zg30

SOLVENT

NS

DS

FIDRES $\quad 8012.820 \mathrm{~Hz}$

RG

DW

DE

TD 1 $2.9999423 \mathrm{sec}$ 45.2 6.50 usec

TDO $2.00000000 \mathrm{sec}$

$=======$ CHANNEL $\mathrm{f} 1 \mathrm{l}=======$ NUC1

P1 15.25 usec PLW1 $12.00000000 \mathrm{~W}$

F2 - Processing parameters

SI $\quad 400.1440000 \mathrm{MHz}$

WDW

SSB
LB

EM

$\mathrm{LB}$
$\mathrm{GB}$
$\mathrm{PC}$

$0.50 \mathrm{~Hz}$

1.00 

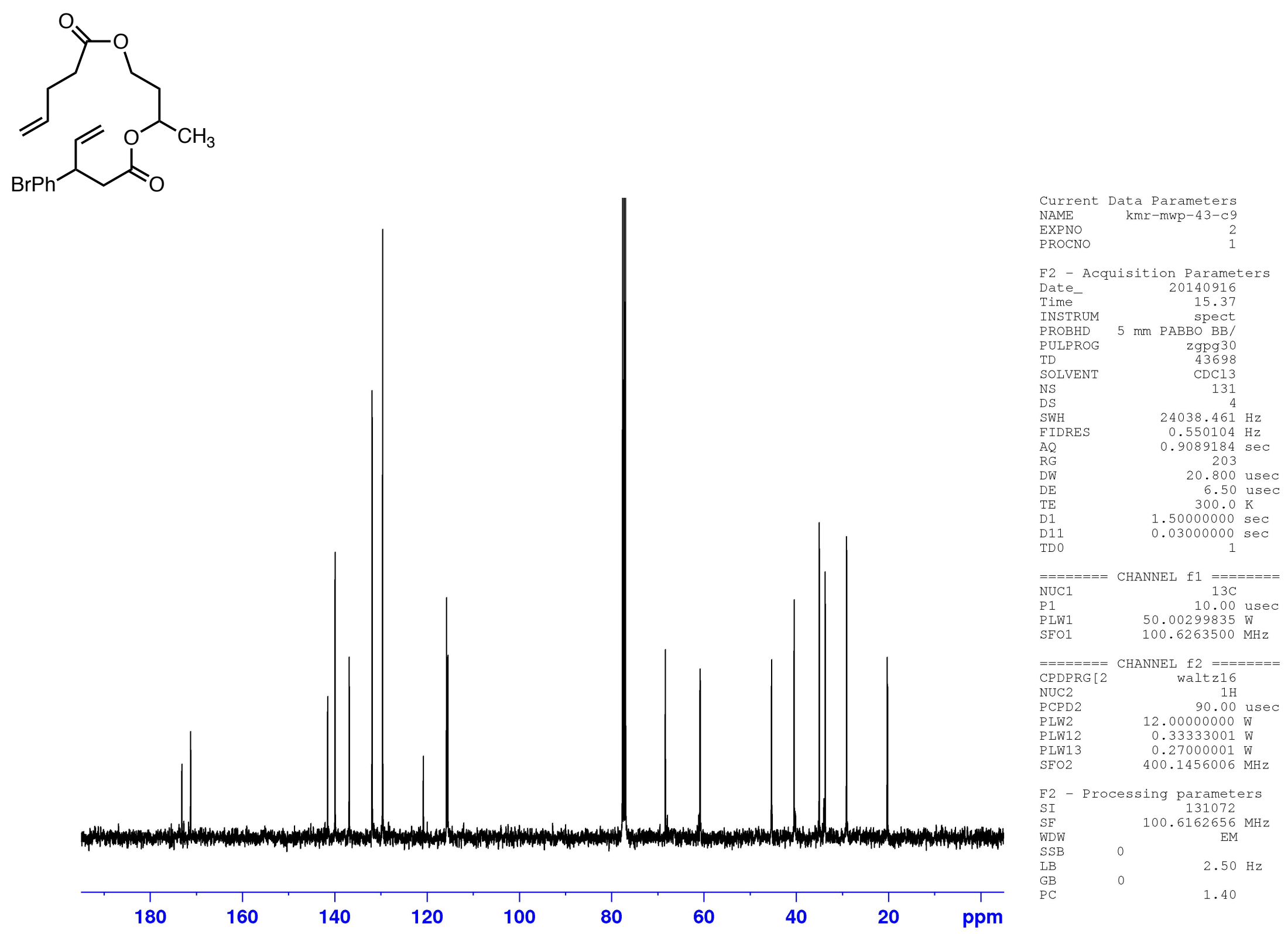
<smiles>C=CCCC(=O)OC(C)CCOC(=O)CC(C=C)c1ccccc1</smiles>
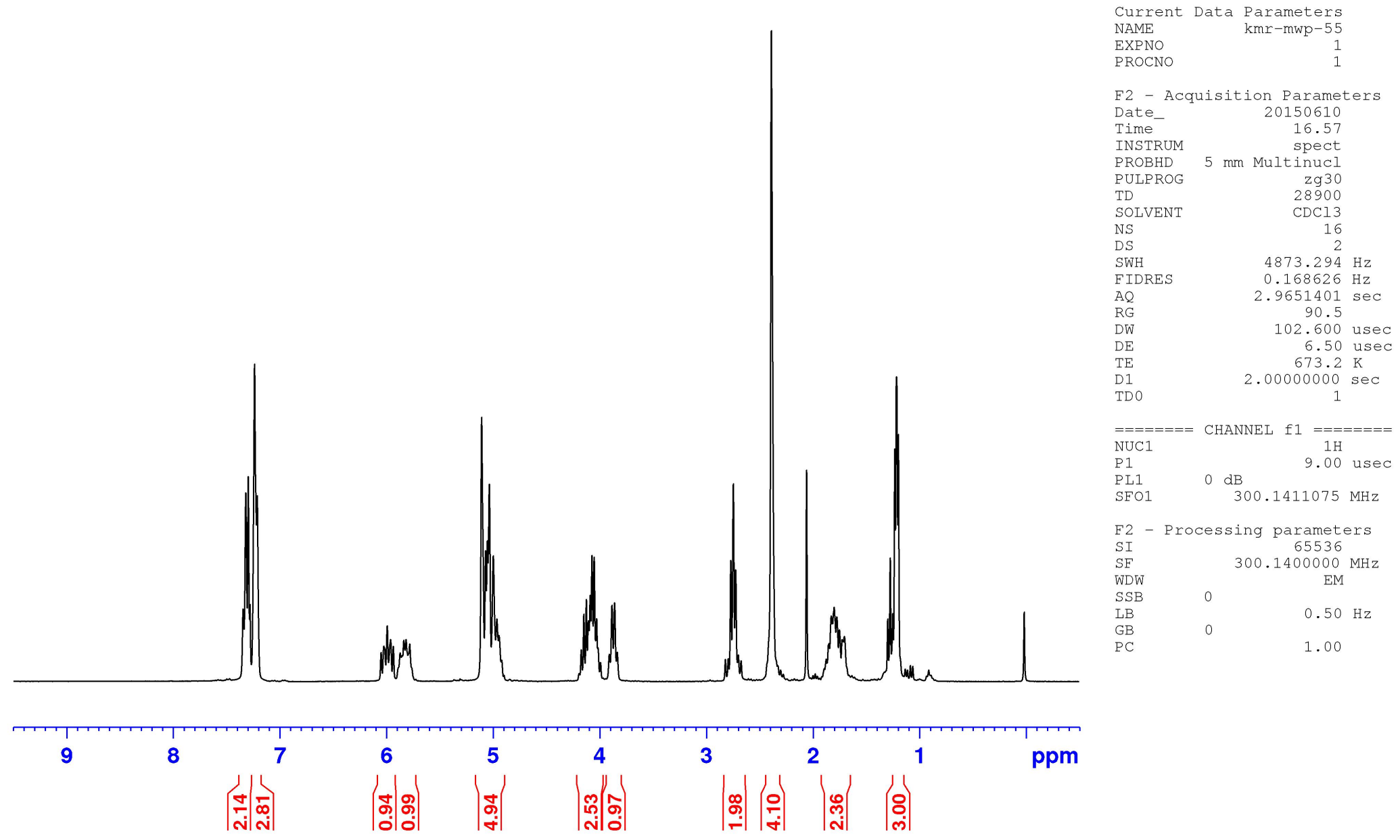

19 
<smiles>C=CCCC(=O)OC(C)CCOC(=O)CC(C=C)c1ccccc1</smiles>
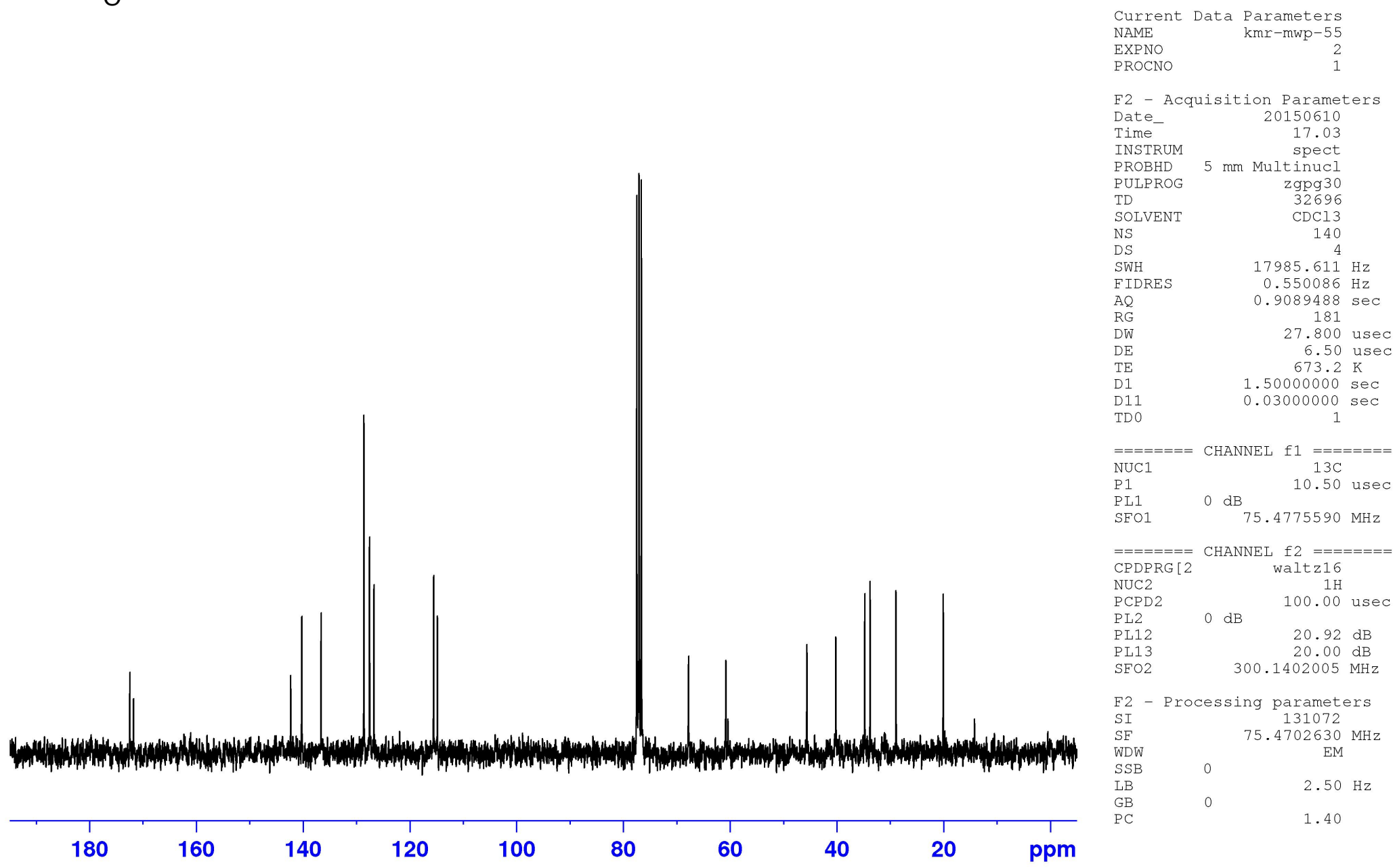

20 

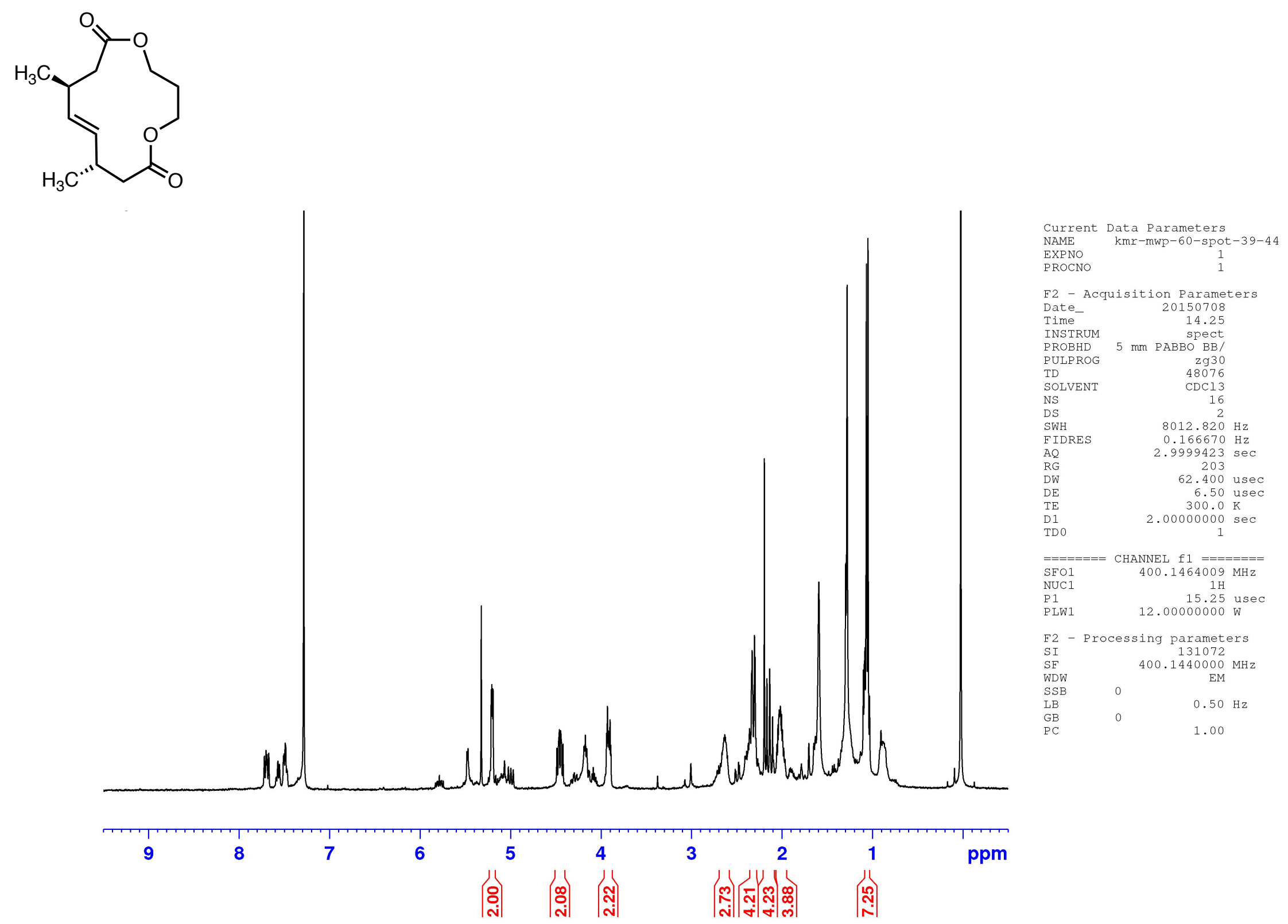
<smiles>CC1/C=C/[C@H](C)CC(=O)OCCCOC(=O)C1</smiles>

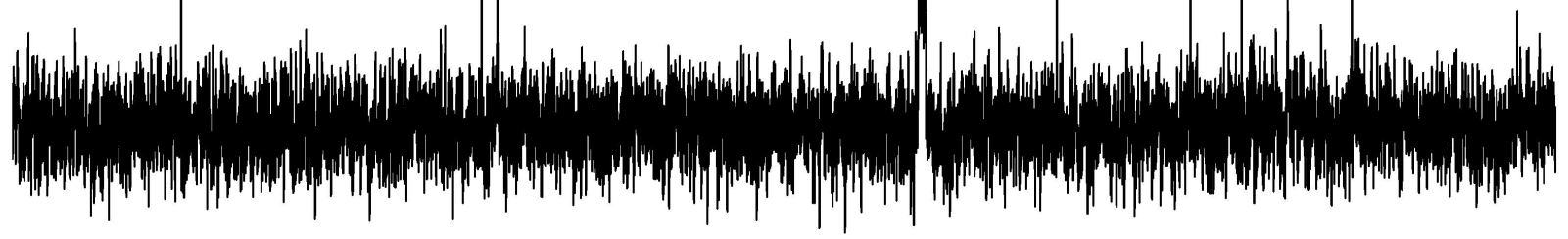
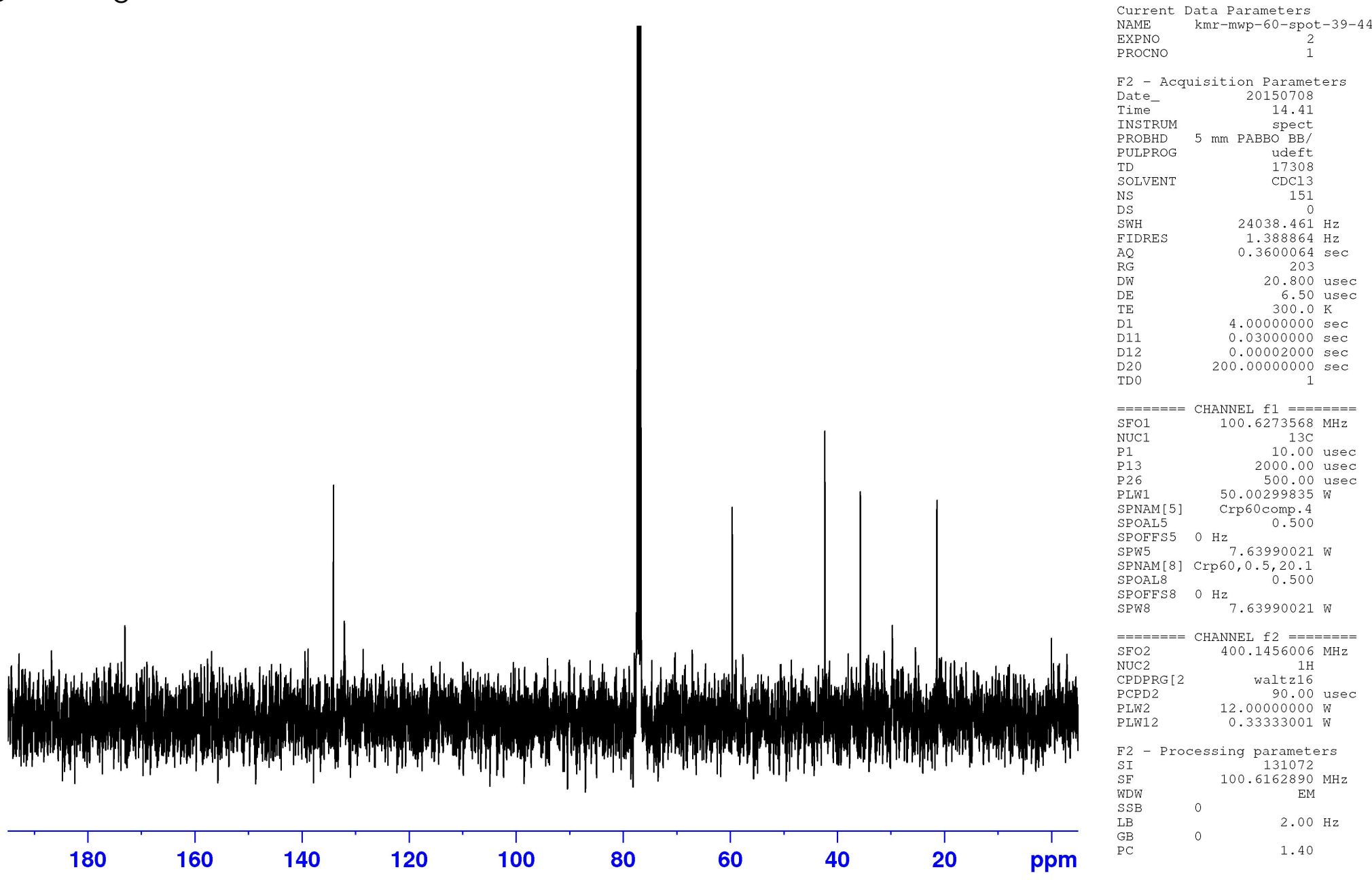

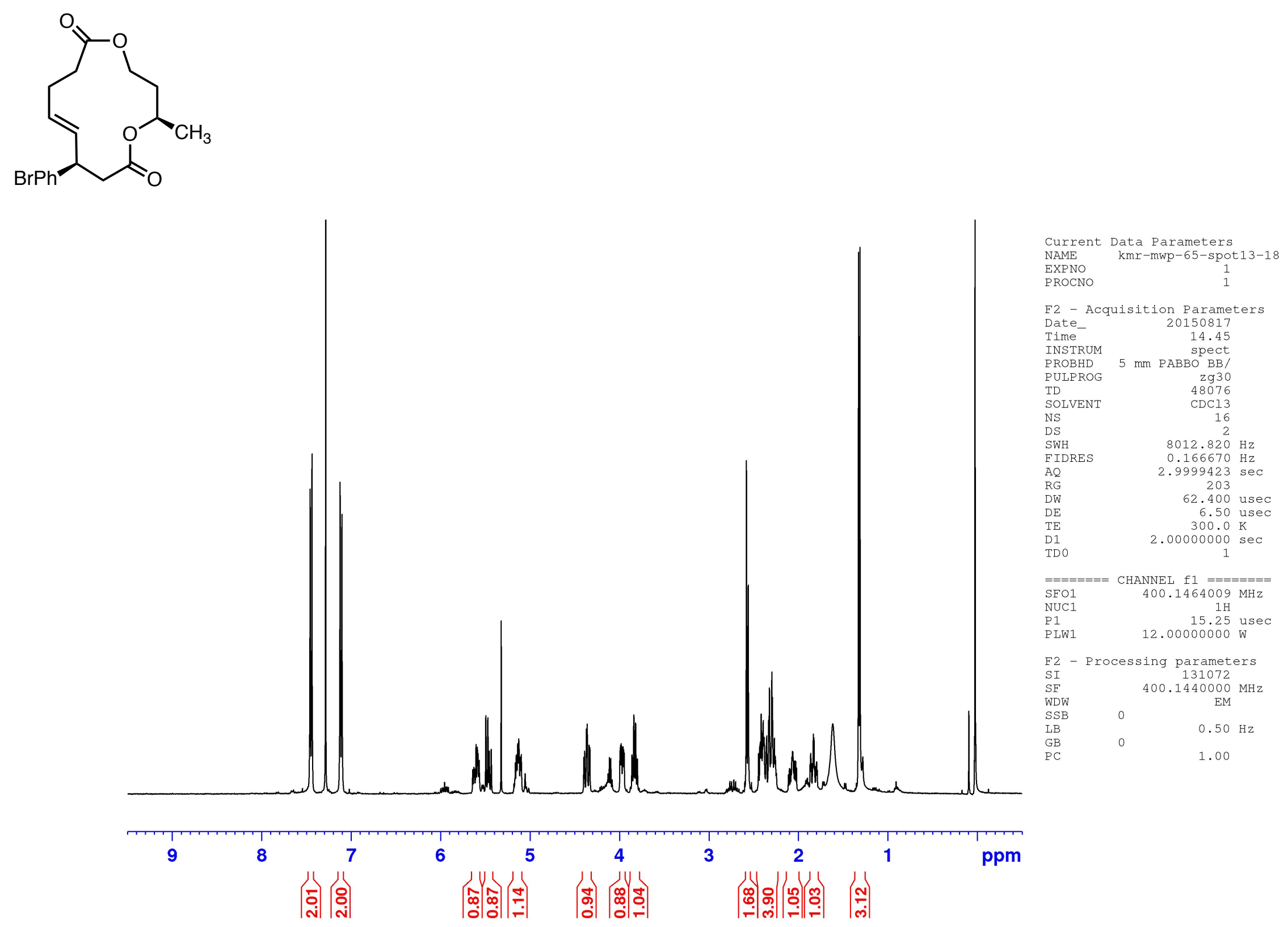

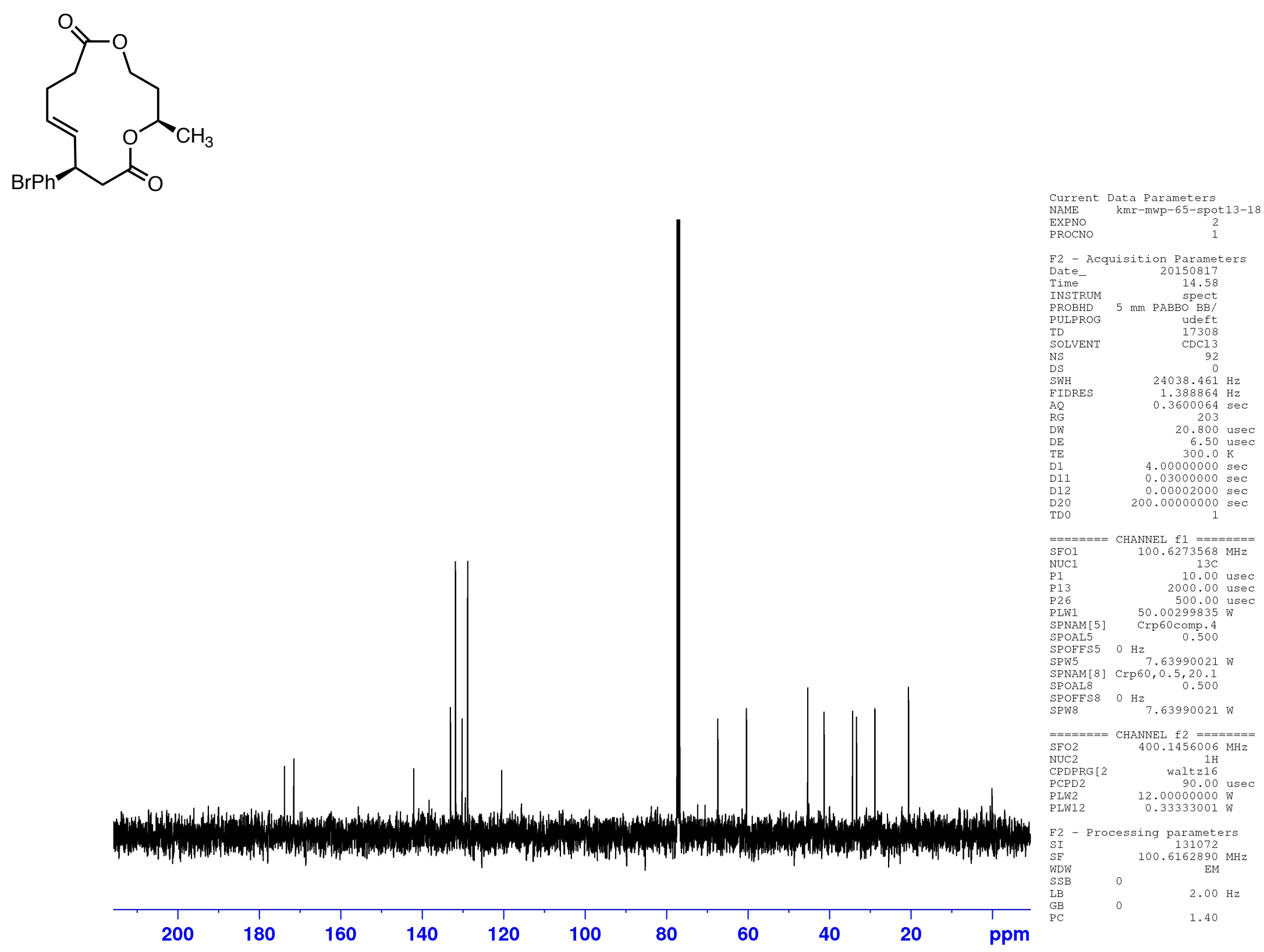

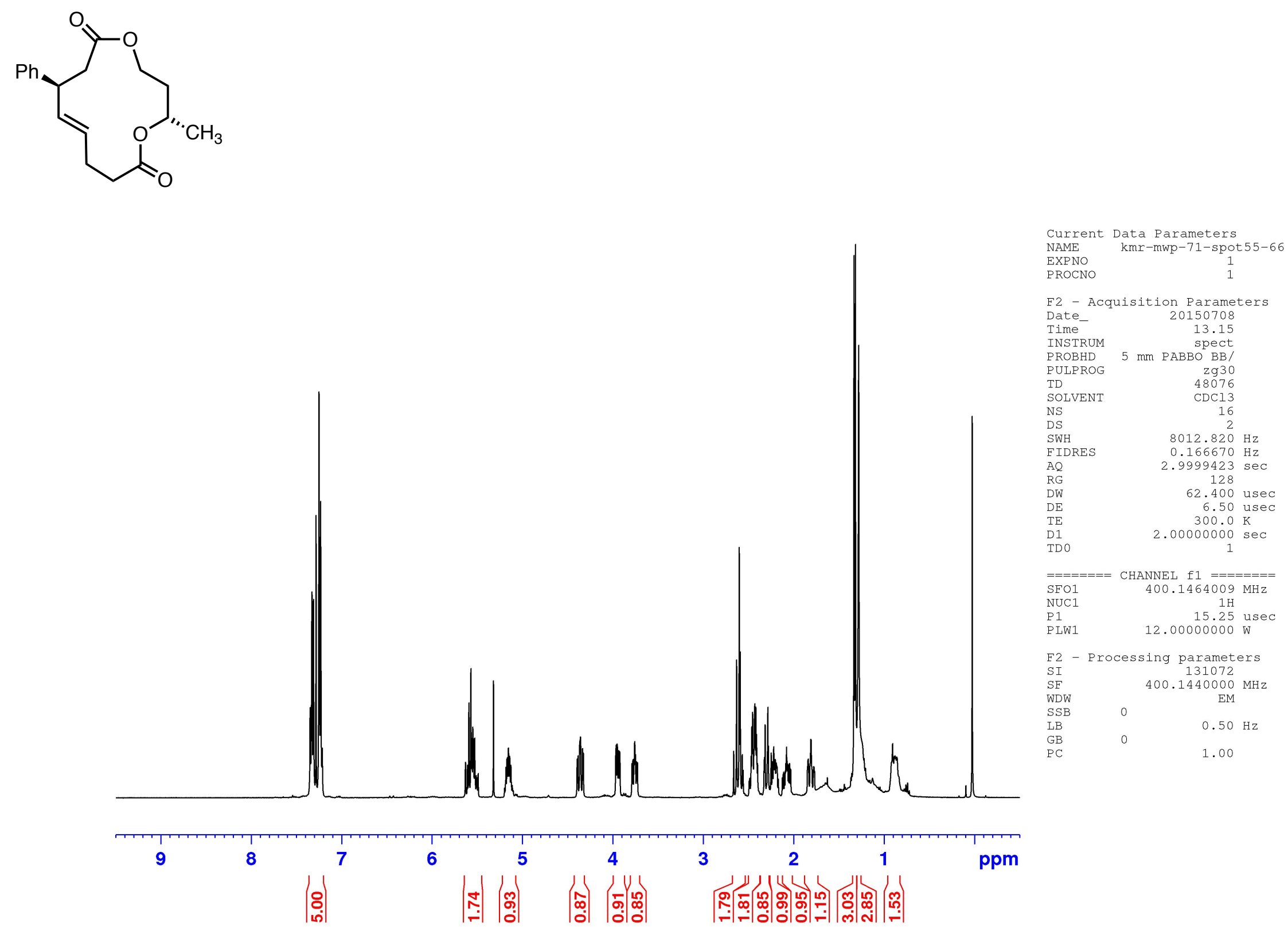

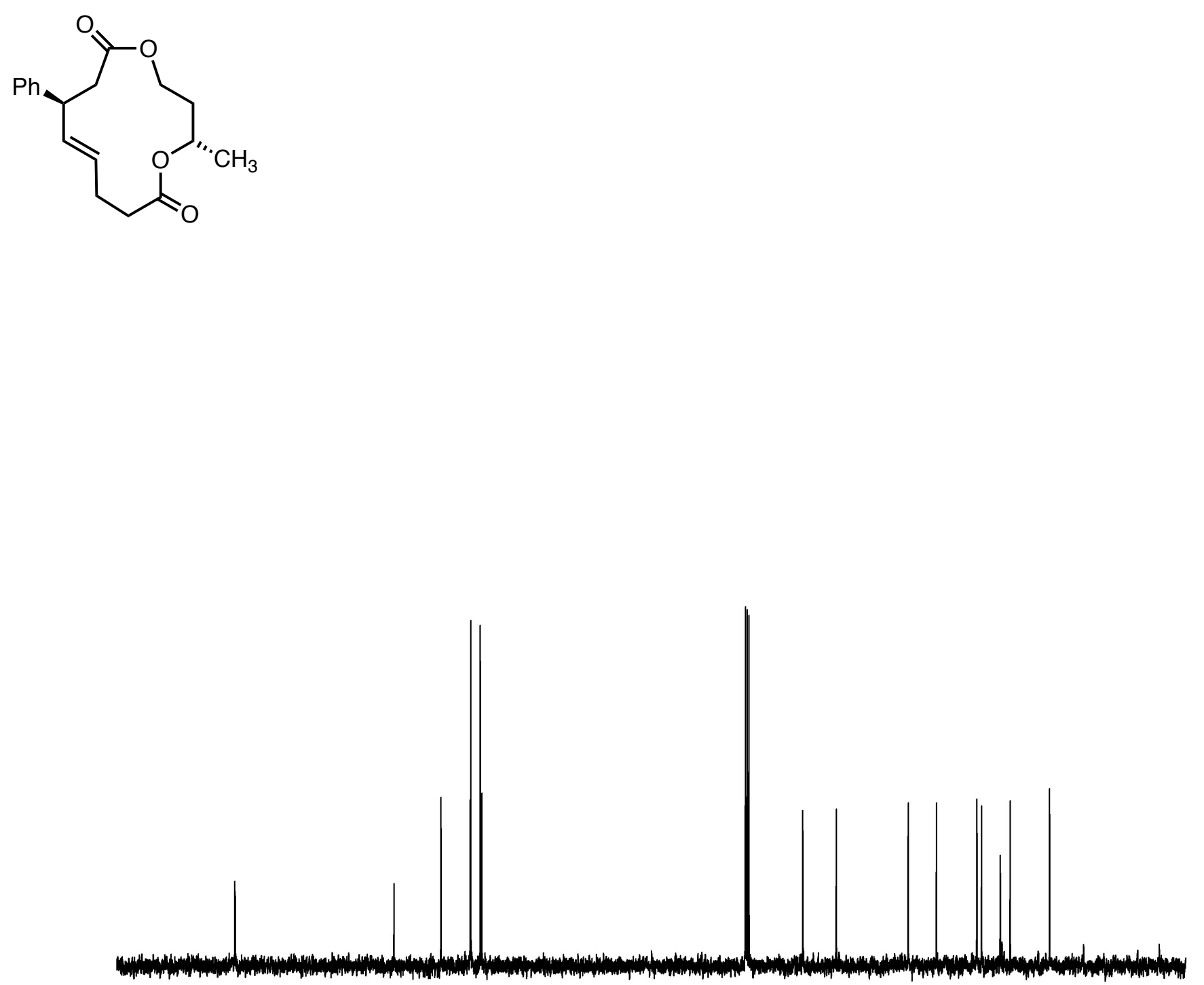

Current Data Parameters
NAME kmr-mwp-71-spot 55-66 EXPNO
PROCNO

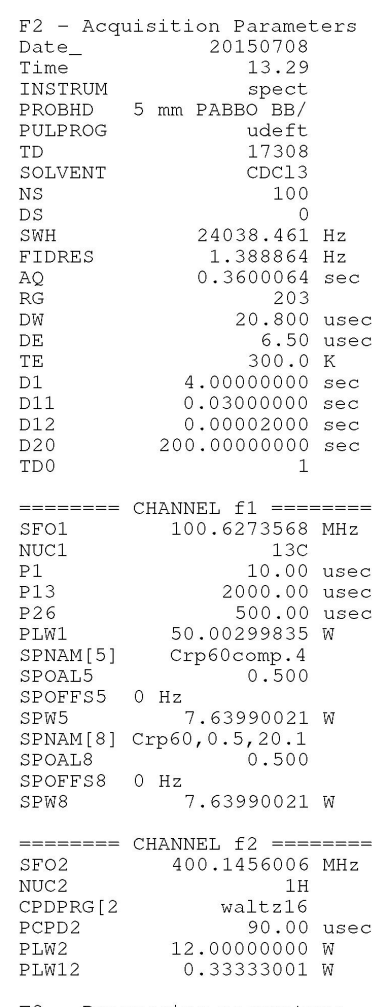

F2 - Processing parameters
SI

$\begin{array}{ll}\text { SI } & 131072 \\ \text { SF } & 100.6162890 \mathrm{MHz}\end{array}$ $\begin{array}{ll}\text { WDW } & \\ \text { SSB } & 0\end{array}$

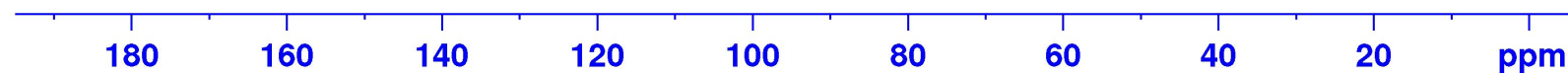

ppm

1.40 